\title{
Sagittal acoustic waves in finite solid-fluid superlattices: Band-gap structure, surface and confined modes, and omnidirectional reflection and selective transmission
}

\author{
Y. El Hassouani, ${ }^{1,2}$ E. H. El Boudouti, ${ }^{1,2, *}$ B. Djafari-Rouhani, ${ }^{2}$ and H. Aynaou ${ }^{1}$ \\ ${ }^{1}$ Département de Physique, Laboratoire de Dynamique et d'Optique des Matériaux, Faculté des Sciences, Université Mohamed I, \\ 60000 Oujda, Morocco \\ ${ }^{2}$ Institut d'Electronique, de Microélectronique et de Nanotechnologie (IEMN), UMR CNRS 8520, UFR de Physique, \\ Université des Sciences et Technologies de Lille, 59655 Villeneuve d'Ascq, France
}

(Received 26 August 2008; published 17 November 2008)

\begin{abstract}
Using a Green's function method, we present a comprehensive theoretical analysis of the propagation of sagittal acoustic waves in superlattices (SLs) made of alternating elastic solid and ideal fluid layers. This structure may exhibit very narrow pass bands separated by large stop bands. In comparison with solid-solid SLs, we show that the band gaps originate both from the periodicity of the system (Bragg-type gaps) and the transmission zeros induced by the presence of the solid layers immersed in the fluid. The width of the band gaps strongly depends on the thickness and the contrast between the elastic parameters of the two constituting layers. In addition to the usual crossing of subsequent bands, solid-fluid SLs may present a closing of the bands, giving rise to large gaps separated by flat bands for which the group velocity vanishes. Also, we give an analytical expression that relates the density of states and the transmission and reflection group delay times in finite-size systems embedded between two fluids. In particular, we show that the transmission zeros may give rise to a phase drop of $\pi$ in the transmission phase, and therefore, a negative delta peak in the delay time when the absorption is taken into account in the system. A rule on the confined and surface modes in a finite SL made of $N$ cells with free surfaces is demonstrated, namely, there are always $N-1$ modes in the allowed bands, whereas there is one and only one mode corresponding to each band gap. Finally, we present a theoretical analysis of the occurrence of omnidirectional reflection in a layered media made of alternating solid and fluid layers. We discuss the conditions for such a structure to exhibit total reflection of acoustic incident waves in a given frequency range for all incident angles. Also, we show how this structure can be used as an acoustic filter that may transmit selectively certain frequencies within the omnidirectional gaps. In particular, we show the possibility of filtering assisted either by cavity modes (in particular sharp Fano resonances) or by interface resonances.
\end{abstract}

DOI: 10.1103/PhysRevB.78.174306

PACS number(s): 43.20. $+\mathrm{g}, 68.35 . \mathrm{Iv}, 62.30 .+\mathrm{d}$

\section{INTRODUCTION}

It is known that the interaction of waves with periodic structures creates interesting interference phenomena. The study of acoustic and electromagnetic waves in periodic layered media has received increased attention during the last two decades due to the unusual physical properties observed in these heterostructures in comparison with bulk materials. ${ }^{1,2}$ The essential property of these structures is the existence of forbidden frequency bands induced by the difference in acoustic and dielectric properties of the constituents and the periodicity of these systems. As concerns acoustic waves, different phononic gaps with appropriate defects have been demonstrated in solid-solid ${ }^{3-19}$ layered structures, leading to several interesting phenomena like in their twodimensional (2D) and three-dimensional (3D) counterpart phononic crystals. ${ }^{20,21}$ Among these phenomena, one can mention (i) omnidirectional band gaps, ${ }^{22,23}$ (ii) the possibility to engineer small-size sonic crystals with locally resonant band gaps in the audible frequency range, ${ }^{24}$ (iii) hypersonic crystals with high-frequency band gaps to enhance acoustooptical interaction ${ }^{25}$ and to realize stimulated emission of acoustic phonons, ${ }^{26}$ and (iv) the possibility to enhance selective transmission through guided modes of a cavity layer inserted in the periodic structure ${ }^{27}$ or by interface resonance modes induced by the superlattice (SL)/substrate interface. ${ }^{28}$
The advantage of one-dimensional (1D) systems lies in the fact that their design is more feasible and they require only relatively simple analytical and numerical calculations. The analytical calculations enable us to understand deeply different physical properties related to the band gaps in such systems.

In comparison with solid-solid layered media, the propagation of acoustic waves in the solid-fluid counterpart structures has received less attention. ${ }^{1}$ The first works on these systems have been carried out by Rytov ${ }^{29}$ and summarized by Brekhovskikh. ${ }^{1}$ Rytov's approach has been used by Schoenberg, ${ }^{30}$ together with propagator matrix formalism, to account for propagation through such a periodic medium in any direction of propagation and at arbitrary frequency. Similar results are also obtained by Rousseau. ${ }^{31}$ In the lowfrequency limit, it was shown ${ }^{30}$ that besides the existence of small gaps, there is one wave speed for propagation perpendicular to the layering and two wave speeds for propagation parallel to the layering which are without analog in solidsolid SLs. The two latter speeds both correspond to compressional waves, and their existence is suggestive of Biot's theory ${ }^{32}$ of wave propagation in porous media. Alternating solid and viscous fluid layers have been proposed recently ${ }^{33}$ as an idealized porous medium to evaluate dispersion and attenuation of acoustic waves in porous solids saturated with fluids. The experimental evidence ${ }^{34}$ of these waves is carried 
out using ultrasonic techniques in Al-water and Plexiglaswater SLs. Also, it was shown theoretically and experimentally that finite-size layered structures composed of a few cells of solid-fluid layers with one s $^{35,36}$ or multiple ${ }^{37}$ periodicity may exhibit large gaps, and the presence of defect layers in these structures may give rise to well-defined defect modes in these gaps. ${ }^{36}$ Recently, ${ }^{38}$ solid layers separated by graded fluid layers have shown the possibility of acoustic Bloch oscillations analogous to the Wannier-Stark ladders of electronic states in a biased SL. ${ }^{39}$ All the above works have mainly dealt with pure longitudinal (compressional) and shear horizontal absorbing waves propagating perpendicular or parallel to the layers. ${ }^{33-38}$

In a recent paper, ${ }^{40}$ we have investigated the propagation and localization of acoustic waves polarized in the sagittal plane, defined by the normal to the surface and the wave vector $k_{\|}$(parallel to the surface), in SLs made of elastic solid and ideal fluid layers. In particular, we have shown the possibility of existence of surface acoustic waves in semiinfinite solid-fluid SL or its interface with a semi-infinite fluid. We have also demonstrated a rule about the existence of surface modes in such structures, namely, by considering together the two complementary semi-infinite SLs obtained by the cleavage of an infinite SL along a plane lying within the fluid layer and parallel to the interfaces, one always has as many localized surface modes as minigaps, for any value of the wave vector $k_{\|}$. However, this rule is not fulfilled when the cleavage is carried out inside the solid layer. Indeed, in this case, the dispersion curves may present zero, one, or two modes inside each gap of the two complementary SLs depending on the position of the plane where the cleavage is produced. Localized and resonant modes associated with the presence of a fluid cap layer with finite or semi-infinite extent-on top of the above-mentioned SLs-have been studied, and different guided modes induced by the adsorbed fluid layer are obtained and their properties are investigated.

In this paper, we are dealing with sagittal acoustic waves in finite-size solid-fluid SLs in contact with one or two semiinfinite fluids on both sides. Our goal is to give closed-form expressions of dispersion relations, densities of states, as well as the transmission and reflection coefficients associated to such systems. These analytical expressions enable us to show peculiar properties related to solid-fluid SLs as compared to solid-solid SLs, namely: (i) The stop bands originate both from the periodicity of the system (Bragg-type gaps) and the transmission zeros induced by the presence of the solid layers immersed in the fluid. The width of the band gaps strongly depends on the thickness and the contrast between the elastic parameters of the two constituting layers. (ii) In addition to the usual crossing of subsequent bands, we show that solid-fluid SLs may present a closing of the bands, giving rise to large gaps separated by flat bands for which the group velocity vanishes. Also, we give an analytical expression that relates the density of states (DOS) and the transmission and reflection group delay times (called also phase times in the literature) in finite-size systems embedded between two fluids. In particular, we show that the transmission zeros may give rise to a phase drop of $\pi$ in the transmission phase and, therefore, a negative delta peak in the delay time when the absorption is taken into account in the system: (iii)
The possibility of the existence of internal resonance induced by a fluid layer and lying at the vicinity of a transmission zero, the so-called Fano resonance.

Finally, we show that similar to solid-solid phononic crystals, layered media made of alternating solid and fluid layers may exhibit total reflection of acoustic incident waves in a given frequency range for all incident angles. In general, this property cannot be fulfilled with a simple finite SL if the incident wave is launched from an arbitrary fluid. Therefore, we propose two solutions to obtain such an omnidirectional band gap, namely, by cladding of the SL with a layer of high acoustic velocities that acts like a barrier for the propagation of phonons or by associating in tandem two different SLs in such a way that the superposition of their band structures exhibits an absolute acoustic band gap. We discuss the appropriate choices of the material and geometrical parameters to realize such structures. The behavior of the transmission coefficients is discussed in relation with the dispersion curves of the finite-size structure. Also, these structures may be used as acoustic filters that may transmit selectively certain frequencies within the omnidirectional gaps. The transmission filtering can be achieved either through the guided modes of a defect layer inserted in the periodic structure or through the interface modes between the SL and a homogeneous fluid medium when these two media are chosen appropriately. These investigations are done within the framework of the Green's function associated to such heterostructures. ${ }^{41}$

The organization of this paper is as follows. Section II presents the model we use for these studies, as well as the analytical results obtained for the Green's function, dispersion relations, transmission and reflection coefficients, and densities of states associated to different solid-fluid layered media. All these quantities represent the ingredients necessary to study analytically and numerically wave propagation in some specific systems such as symmetric and asymmetric finite SLs (Secs. III and IV) and omnidirectional reflection and selective transmission in such systems (Secs. V and VI). The main conclusions are summarized in Sec. VII.

\section{INTERFACE RESPONSE THEORY OF CONTINUOUS MEDIA}

\section{A. Overview}

In this paper, we are interested in the propagation of acoustic waves in composite materials composed of elastic solid and ideal fluid layers. The waves are polarized in the sagittal plane defined by the normal to the interfaces $\left(x_{3} \mathrm{di}\right.$ rection) and the wave vector $k_{\|}$(parallel to the interfaces). We choose $k_{\|}$along the $x_{1}$ direction. It is worth noticing that the assumption of ideal fluid behavior is valid over a very broad frequency range for which the viscous skin depth $\sigma$ $=(2 \eta / \rho \omega)$ is much smaller than the fluid layer thickness $d_{f}$ ( $\eta$ and $\rho$ are the viscosity and the density of the fluid). This study is performed with the help of the interface response theory ${ }^{41}$ of continuous media, which permits us to calculate the Green's function of any composite material. In what follows, we present the basic concept and the fundamental equations of this theory. ${ }^{41}$ 
Let us consider any composite material contained in its space of definition $D$ and formed out of $N$ different homogeneous pieces located in their domains $D_{i}$. Each piece is bounded by an interface $M_{i}$, adjacent in general to $j(1 \leq j$ $\leq J$ ) other pieces through subinterfaces domains $M_{i j}$. The ensemble of all these interface spaces $M_{i}$ will be called the interface space $M$ of the composite material.

The elements of the Green's function $g(D D)$ of any composite material can be obtained from ${ }^{41}$

$$
\begin{aligned}
g(D D)= & G(D D)-G(D M) G^{-1}(M M) G(M D) \\
& +G(D M) G^{-1}(M M) g(M M) G^{-1}(M M) G(M D),
\end{aligned}
$$

where $G(D D)$ is the Green's function of a reference continuous medium and $g(M M)$ is the interface element of the Green's function of the composite system. Here all the Green's functions are those associated to the displacement field. For instance, this means that the bulk Green's function $G(D D)$ of elastic solid and viscous fluid media can be derived from the equation of motion of displacement fields (the details of the calculations for the derivation of these expressions are given in Ref. 41). The inverse $[g(M, M)]^{-1}$ of $g(M M)$ is obtained for any point in the space of the interface $M=\cup M_{i}$ as a superposition of the different $g_{i}^{-1}\left(M_{i}, M_{i}\right),{ }^{41}$ inverse of the $g_{i}\left(M_{i}, M_{i}\right)$ for each constituent $i$ of the composite system. The latter quantities are given by the equation

$$
g_{i}^{-1}\left(M_{i}, M_{i}\right)=\Delta_{i}\left(M_{i}, M_{i}\right) G_{i}^{-1}\left(M_{i}, M_{i}\right),
$$

where

$$
\Delta_{i}\left(M_{i}, M_{i}\right)=I\left(M_{i}, M_{i}\right)+A_{i}\left(M_{i}, M_{i}\right),
$$

( $I$ is the unit matrix) and

$$
A_{i}\left(X, X^{\prime}\right)=\left.V_{c}\left(X^{\prime \prime}\right) G_{i}\left(X^{\prime \prime}, X^{\prime}\right)\right|_{X^{\prime \prime}=X},
$$

where $X, X^{\prime \prime} \in D_{i}$.

In Eq. (4), the cleavage operator $V_{c}$ acts only in the surface domain $M_{i}$ of $D_{i}$ and cuts the finite-size or semi-infinitesize block out of the infinite homogeneous medium. ${ }^{41} A_{i}$ is called the surface response operator of block $i$.

The new interface states can be calculated from ${ }^{41}$

$$
\operatorname{det}\left[g^{-1}(M M)\right]=0
$$

or equivalently

$$
\operatorname{det}[\Delta(M M)]=0,
$$

showing that if one is interested in calculating the interface states of a composite, one only needs to know the inverse of the Green's function of each individual block in the space of their respective surfaces and/or interfaces.

Moreover, if $U(D)$ represents an eigenvector of the reference system, Eq. (1) enables one to calculate the eigenvectors $u(D)$ of the composite material ${ }^{42}$

$$
\begin{aligned}
u(D)= & U(D)-U(M) G^{-1}(M M) G(M D) \\
& +U(M) G^{-1}(M M) g(M M) G^{-1}(M M) G(M D)
\end{aligned}
$$

In Eq. (7), $U(D), U(M)$, and $u(D)$ are row vectors. Equation (7) enables one also to calculate all the waves reflected and transmitted by the interfaces, as well as the reflection and the transmission coefficients of the composite system. In this case, $U(D)$ must be replaced by a bulk wave launched in one homogeneous piece of the composite material. ${ }^{42}$

\section{B. Inverse surface Green's functions of the elementary constituents}

Before addressing the problem of the fluid-solid SL, it is helpful to know the surface elements of its elementary constituents, namely, the Green's function of an ideal fluid of thickness $d_{f}$, sound speed $v_{f}$, and mass density $\rho_{f}$; and an elastic isotropic solid characterized by its thickness $d_{s}$, longitudinal speed $v_{\ell}$, transverse speed $v_{t}$, and mass density $\rho_{s}$. Let us first notice that the Green's functions associated with sagittal waves in an elastic solid is a $4 \times 4$ matrix as these waves exhibit two directions of vibrations $s^{40,43}$ in the sagittal plane $\left(x_{1}, x_{3}\right)$. However, the ideal fluid layer is characterized by only one degree of vibration, and its $4 \times 4$ Green's function matrix has only $x_{3} x_{3}$ nonzero elements. Therefore, as far as we are interested by solid-fluid interfaces, the $4 \times 4$ Green's function of the solid layer can be reduced to a 2 $\times 2$ matrix by keeping only the $x_{3} x_{3}$ components (see Ref. 40 for more details). In addition, the calculations of the dispersion relations, reflection, and transmission coefficients (see below) can be deduced only from the knowledge of the $x_{3} x_{3}$ components of the Green's functions. That is why in what follows, we shall be interested only by the calculation of the $x_{3} x_{3}$ components of the different Green's functions.

The inverse Green's function of an ideal fluid layer in the space of the two surfaces of the layer is given by ${ }^{40}$

$$
\left[g_{f}(M M)\right]^{-1}=\left(\begin{array}{ll}
a & b \\
b & a
\end{array}\right),
$$

where

$$
\begin{gathered}
a=-F \frac{C_{f}}{S_{f}}, \quad b=\frac{F}{S_{f}}, \\
C_{f}=\cosh \left(\alpha_{f} d_{f}\right), \quad S_{f}=\sinh \left(\alpha_{f} d_{f}\right), \\
F=-\rho_{f} \frac{\omega^{2}}{\alpha_{f}} \quad \text { and } \quad \alpha_{f}=j\left(\frac{\omega^{2}}{v_{f}^{2}}-k_{\|}^{2}\right)^{1 / 2},
\end{gathered}
$$

and the inverse Green's function of the elastic solid layer in the space of the two surfaces of the layer is ${ }^{40}$

$$
\left[g_{s}(M M)\right]^{-1}=\left(\begin{array}{ll}
A & B \\
B & A
\end{array}\right),
$$

where

$$
\begin{gathered}
A=-\gamma \frac{C_{\ell}}{S_{\ell}}-\beta \frac{C_{t}}{S_{t}}, \quad B=\frac{\gamma}{S_{\ell}}+\frac{\beta}{S_{t}}, \\
\gamma=-\rho \frac{v_{t}^{4}}{\omega^{2} \alpha_{\ell}}\left(k_{\|}^{2}+\alpha_{t}^{2}\right)^{2}, \quad \beta=4 \rho \frac{v_{t}^{4}}{\omega^{2}} \alpha_{t} k_{\|}^{2},
\end{gathered}
$$




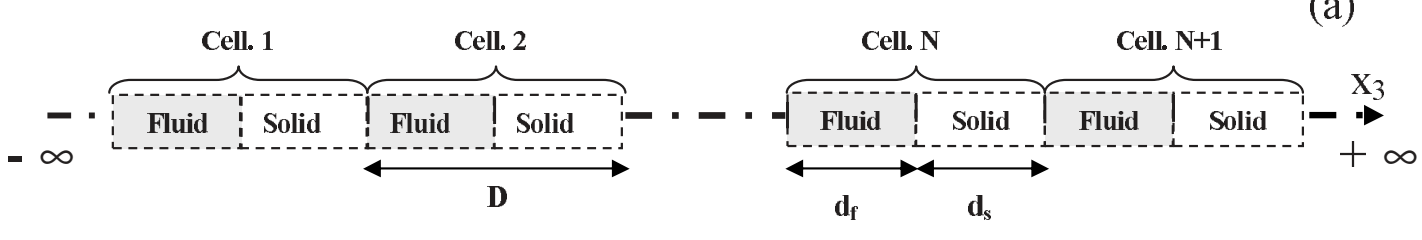

(b)

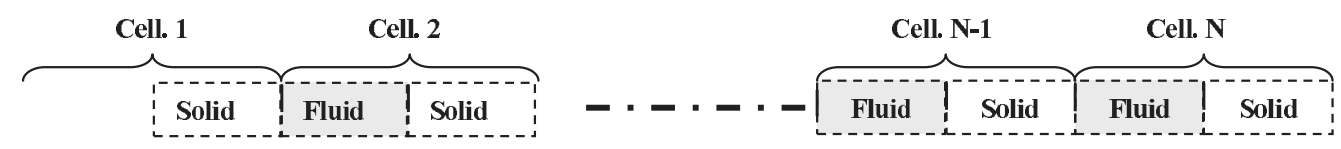

(c)
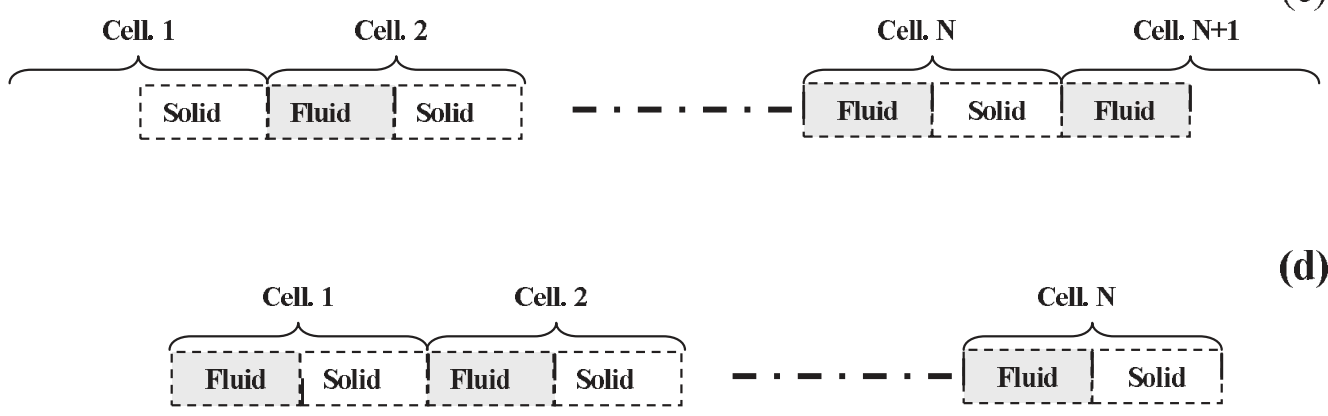

(d)

(e)

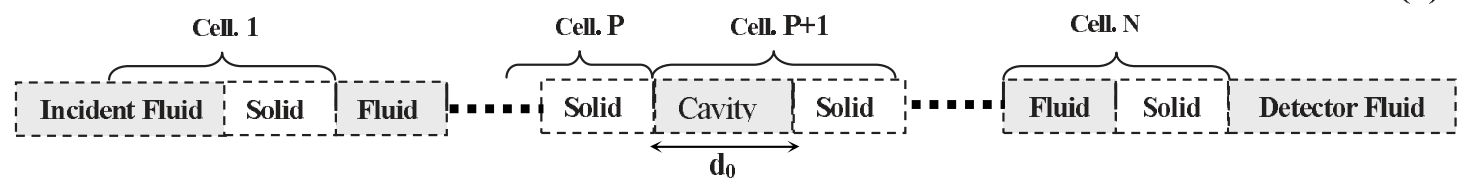

FIG. 1. (a) Schematic representation of an infinite solid-fluid superlattice (SL). $d_{f}$ and $d_{s}$ are the thicknesses of the fluid and solid layers, respectively. $D=d_{f}+d_{s}$ is the period of the SL. (b) Schematic representation of a finite SL composed of $N$ cells with (solid, solid) terminations on both sides. (c), (d) The same as (b) but for a SL with (solid, fluid) and (fluid, solid) terminations, respectively. The two extremities are free of stress. (e) Schematic representation of a finite SL with a cavity fluid layer in the cell $p+1$. The whole system is embedded between two semi-infinite fluids.

$$
\begin{aligned}
C_{t}=\cosh \left(\alpha_{t} d_{s}\right), \quad C_{\ell}=\cosh \left(\alpha_{\ell} d_{s}\right), \quad S_{t}=\sinh \left(\alpha_{t} d_{s}\right), \\
S_{\ell}=\sinh \left(\alpha_{\ell} d_{s}\right),
\end{aligned}
$$

and

$$
\alpha_{t}^{2}=k_{\|}^{2}-\frac{\omega^{2}}{v_{t}^{2}}, \quad \alpha_{\ell}^{2}=k_{\|}^{2}-\frac{\omega^{2}}{v_{\ell}^{2}} .
$$

The inverse Green's function of a semi-infinite fluid and a semi-infinite solid with free surfaces are given, respectively, by

$$
g_{f}^{-1}(0,0)=-F
$$

and

$$
g_{s}^{-1}(0,0)=-\gamma-\beta,
$$

where $F$ is defined by Eq. (9c) and $\gamma$ and $\beta$ are given by Eq. (12).

\section{Surface Green's function of an infinite solid-fluid superlattice}

Let us emphasize that in the geometry of the structures studied, all the interfaces are taken to be parallel to $\left(x_{1}, x_{2}\right)$ plane. A space position along the $x_{3}$ axis in medium $i$ belonging to the unit cell $n$ is indicated by $\left(n, i, x_{3}\right)$, where $-d_{i} / 2$ $<x_{3}<d_{i} / 2[i=f$ for the fluid and $i=s$ for the solid, see Fig. 1(a)]. As we are interested by the propagation of sagittal acoustic waves in such structures, the elements of the Green's functions take the form $g\left(\omega^{2}, k_{\|} \mid n, i, x_{3} ; n^{\prime}, i^{\prime}, x_{3}^{\prime}\right)$, where $\omega$ is the frequency of the acoustic wave and $k_{\|}$is the wave vector parallel to the interfaces. For the sake of simplicity, we shall omit in the following the parameters $\omega^{2}$ and $k_{\|}$, and we note as $g\left(n, i, x_{3} ; n^{\prime}, i^{\prime}, x_{3}^{\prime}\right)$ the $x_{3} x_{3}$ component of the Green's function.

The Green's function of the infinite SL [Fig. 1(a)] in the space of interfaces is obtained by a linear juxtaposition of the $2 \times 2$ matrices [Eqs. (8) and (10)] at the different interfaces, leading to a tridiagonal matrix. 
Taking advantage of the periodicity $D$ in the direction $x_{3}$ of the solid-fluid SL, the Fourier transformed $\left[g\left(k_{3} ; M, M\right)\right]^{-1}$ of the above infinite tridiagonal matrix within one unit cell $(1 \leq i \leq N)$ has the following form: ${ }^{3}$

$$
\left[g\left(k_{3} ; M M\right)\right]^{-1}=\left(\begin{array}{cc}
A+a & B+b e^{-j k_{3} D} \\
B+b e^{j k_{3} D} & A+a
\end{array}\right) .
$$

The bulk bands (eigenmodes) of the infinite solid-fluid SL are easily obtained from Eqs. (5) and (17) in the following form:

$$
\cos \left(k_{3} D\right)=\frac{A^{2}-B^{2}+a^{2}-b^{2}+2 A a}{2 B b}=\eta,
$$

where $k_{3}$ is the component perpendicular to the slabs of the propagation vector $\vec{k} \equiv\left(k_{\|}, k_{3}\right)$.

It is also straightforward to Fourier analyze back into real space all the elements of $g\left(k_{3} ; M M\right)$ and obtain all the interface elements of $g$ in the following form: ${ }^{3}$

$$
\begin{aligned}
g\left(n, f,-\frac{d_{f}}{2} ; n^{\prime}, f,-\frac{d_{f}}{2}\right) & =g\left(n, f, \frac{d_{f}}{2} ; n^{\prime}, f, \frac{d_{f}}{2}\right) \\
& =-\frac{(A+a)}{B b} \frac{t^{\left|n-n^{\prime}\right|+1}}{t^{2}-1}, \\
g\left(n, f,-\frac{d_{f}}{2} ; n^{\prime}, f, \frac{d_{f}}{2}\right) & =-\frac{t^{\left|n-n^{\prime}\right|+1}}{B\left(t^{2}-1\right)}+\frac{t^{\left|n-n^{\prime}-1\right|+1}}{b\left(t^{2}-1\right)}, \\
g\left(n, f, \frac{d_{f}}{2} ; n^{\prime}, f,-\frac{d_{f}}{2}\right) & =-\frac{t^{\left|n-n^{\prime}\right|+1}}{B\left(t^{2}-1\right)}+\frac{t^{\left|n-n^{\prime}+1\right|+1}}{b\left(t^{2}-1\right)} .
\end{aligned}
$$

In this expressions, $t$ represents $e^{i k_{3} D}$ and is defined by ${ }^{3}$

$$
\begin{aligned}
& t=\eta+\sqrt{\eta^{2}-1} \quad \text { if } \quad \eta<-1, \\
& t=\eta+i \sqrt{1-\eta^{2}} \quad \text { if } \quad|\eta| \leq 1, \\
& t=\eta-\sqrt{\eta^{2}-1} \quad \text { if } \quad \eta>-1 .
\end{aligned}
$$

\section{Inverse surface Green's functions of finite solid-fluid superlattices with free surfaces}

We consider in this subsection different finite-size solidfluid SLs with free surfaces. The surface layers on both ends of these systems could be (solid, solid) [Fig. 1(b)], (solid, fluid) [Fig. 1(c)], or (fluid, solid) [Fig. 1(d)]. The knowledge of the inverse of the Green's functions on both ends of these systems constitutes the necessary ingredients to deduce easily the dispersion relations, as well as the transmission and reflection coefficients through different finite-size solid-fluid SLs with or without defect layers. In what follows, we shall detail the results concerning the Green's function calculation of the structure depicted in Fig. 1(b) with (solid, solid) terminations and give briefly the results concerning the other structures in Figs. 1(c) and 1(d) with (solid, fluid) and (fluid, solid) terminations, respectively.

The structure in Fig. 1(b) is constructed from the infinite SL of Fig. 1(a). In a first step, one suppresses the fluid layers in the cells $n=1$ and $n=N+1$. For this new system composed of a finite SL and two semi-infinite SLs on both sides (not shown here), the inverse surface Green's function, $\left[g_{s}(M, M)\right]^{-1}$, is an infinite tridiagonal matrix defined in the interface domain of all the sites $n,(-\infty \leq n \leq+\infty)$. The matrix is similar to the one associated with the infinite SL. Only a few matrix elements differ, namely, those associated with the interface space $M_{s}=\left\{\left(n=1, i=f,-\frac{d_{f}}{2}\right),(n=1, i\right.$ $\left.\left.=f, \frac{d_{f}}{2}\right),\left(n=N+1, i=f,-\frac{d_{f}}{2}\right),\left(n=N+1, i=f, \frac{d_{f}}{2}\right)\right\}$. The cleavage operator

$$
V_{c}(M M)=\left[g_{s}(M, M)\right]^{-1}-[g(M, M)]^{-1}
$$

is the following $4 \times 4$ square matrix defined in the interface domain $M_{s}$ :

$$
V_{c}\left(M_{s} M_{s}\right)=\left(\begin{array}{cccc}
-a & -b & 0 & 0 \\
-b & -a & 0 & 0 \\
0 & 0 & -a & -b \\
0 & 0 & -b & -a
\end{array}\right) .
$$

In addition, using Eq. (19) one can write the elements of the surface Green's function of the infinite SL in the interface space $M_{s}$ in the form of a $4 \times 4$ square matrix

$$
g\left(M_{s} M_{s}\right)=\frac{t}{t^{2}-1}\left(\begin{array}{cccc}
-\frac{A+a}{B b} & \frac{b+B t}{B b} & -\frac{A+a}{B b} t^{N} & \frac{b+B t}{B b} t^{N} \\
\frac{b+B t}{B b} & -\frac{A+a}{B b} & \frac{B+b t}{B b} t^{N-1} & -\frac{A+a}{B b} t^{N} \\
-\frac{A+a}{B b} t^{N} & \frac{B+b t}{B b} t^{N-1} & -\frac{A+a}{B b} & \frac{b+B t}{B b} \\
\frac{b+B t}{B b} t^{N} & -\frac{A+a}{B b} t^{N} & \frac{b+B t}{B b} & -\frac{A+a}{B b}
\end{array}\right),
$$




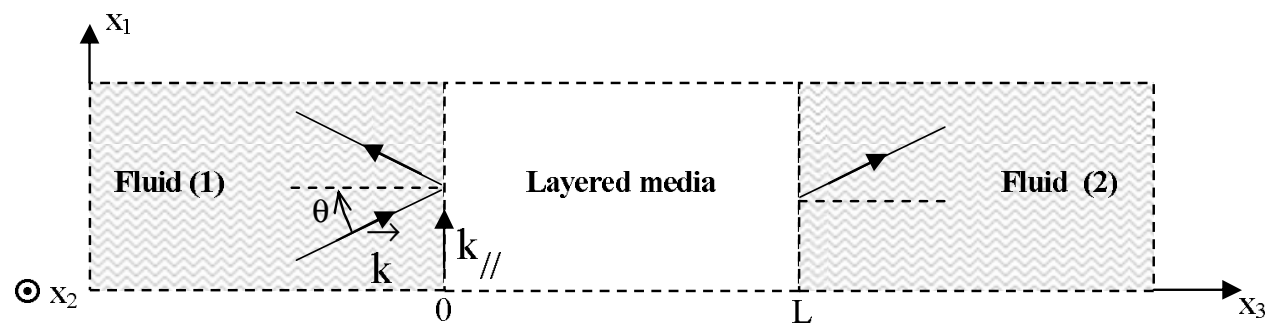

FIG. 2. Schematic representation of a finite layered structure inserted between two different semi-infinite fluids labeled 1 and $2 . k_{\|}$is the component of the wave vector $k$ parallel to the layers. $\theta$ is the incident angle in fluid 1 .

where $a, b, A$, and $B$ are defined by Eqs. (9) and (11).

Using Eqs. (22) and (23), one obtains the matrix operator $\Delta\left(M_{s} M_{s}\right)=I\left(M_{s} M_{s}\right)+V_{c}\left(M_{s} M_{s}\right) g\left(M_{s} M_{s}\right)$ in space $M_{s}$. For the calculation of the inverse Green's function on both ends of the structure in Fig. 1(b), we only need the matrix $\Delta\left(M_{0} M_{0}\right), \quad$ where $M_{0}=\left\{\left(n=1, i=f, \frac{d_{f}}{2}\right),(n=N+1, i=f\right.$, $\left.\left.-\frac{d_{f}}{2}\right)\right\}$ represents the interface space corresponding to both extremities of the system in Fig. 1(b),

$$
\Delta\left(M_{0} M_{0}\right)=\left(\begin{array}{cc}
1-\frac{t}{t^{2}-1} \frac{Y_{1}}{B b} & -\frac{t^{N}}{t^{2}-1} \frac{Y_{2}}{B b} \\
-\frac{t^{N}}{t^{2}-1} \frac{Y_{2}}{B b} & 1-\frac{t}{t^{2}-1} \frac{Y_{1}}{B b}
\end{array}\right),
$$

where $Y_{1}=b^{2}-a^{2}-a A+B b t$ and $Y_{2}=a B-A b t$.

The inverse of the surface Green's function $d_{s s}^{-1}\left(M_{0} M_{0}\right)$ in the interface space $M_{0}$ of the finite SL in Fig. 1(b) is given by Eq. (2),

$$
d_{s s}^{-1}\left(M_{0} M_{0}\right)=\Delta\left(M_{0} M_{0}\right) g^{-1}\left(M_{0} M_{0}\right)
$$

with

$$
g\left(M_{0} M_{0}\right)=\frac{t}{t^{2}-1}\left(\begin{array}{cc}
-\frac{A+a}{B b} & \frac{B+b t}{B b} t^{N-1} \\
\frac{B+b t}{B b} t^{N-1} & -\frac{A+a}{B b}
\end{array}\right) .
$$

From Eqs. (24)-(26), one obtains finally

$$
d_{s s}^{-1}\left(M_{0} M_{0}\right)=\left(\begin{array}{ll}
A(N) & B(N) \\
B(N) & A(N)
\end{array}\right),
$$

with

$$
\begin{gathered}
A(N)=\left(\frac{Y_{1}}{A+a}\right)\left[1-B b\left(t-\frac{1}{t}\right) \frac{Y_{1}}{\Delta}\right], \\
B(N)=B b\left(t-\frac{1}{t}\right) \frac{Y_{1} Y_{2}}{(A+a) \Delta} t^{N-1},
\end{gathered}
$$

and

$$
\Delta=Y_{1}^{2}-Y_{2}^{2} t^{2(N-1)} .
$$

By following the same procedure and after some algebraic calculations, one can obtain, respectively, the operator $\Delta\left(M_{0}^{\prime} M_{0}^{\prime}\right)$, and the inverse Green's function $d_{s f}^{-1}\left(M_{0}^{\prime} M_{0}^{\prime}\right)$ in the interface space $M_{0}^{\prime}=\left\{\left(n=1, i=f, \frac{d_{f}}{2}\right),(n=N+1, i=f\right.$, $\left.\left.-\frac{d_{f}}{2}\right)\right\}$ of the structure depicted in Fig. 1(c) ended at the left side by a solid layer and at the right side by a fluid layer, namely,

$$
\stackrel{\overleftrightarrow{\Delta}\left(M_{0}^{\prime} M_{0}^{\prime}\right)}{=\left(\begin{array}{cc}
1-\frac{t}{t^{2}-1} \frac{Y_{1}}{B b} & -\frac{t^{N+1}}{t^{2}-1} \frac{Y_{1}}{B b} \\
\frac{t^{N+1}}{t^{2}-1}\left[t+\frac{1}{t}+\frac{Y_{1}}{B b}\right] & 1-\frac{t}{t^{2}-1}\left[t+\frac{1}{t}+\frac{Y_{1}}{B b}\right]
\end{array}\right)}
$$

and

$$
d_{s f}^{-1}\left(M_{0}^{\prime} M_{0}^{\prime}\right)=\left(\begin{array}{ll}
X(N) & Y(N) \\
Y(N) & Z(N)
\end{array}\right)
$$

where

$$
\begin{gathered}
X(N)=\left[-\frac{B b}{(A+a)\left(1-t^{2 N}\right)}\right]\left[t-\frac{1}{t}-\frac{Y_{1}}{B b}\left(1-t^{2 N}\right)\right], \\
Y(N)=\left[-\frac{B b}{(A+a)\left(1-t^{2 N}\right)}\right]\left(t-\frac{1}{t}\right) t^{N},
\end{gathered}
$$

and

$$
Z(N)=\left[\frac{B b}{(A+a)\left(1-t^{2 N}\right)}\right]\left[2 t+\frac{Y_{1}}{B b}\left(1-t^{2 N}\right)\right] .
$$

Now, if the structure is ended by a fluid layer on the left side and a solid layer on the right side [Fig. 1(d)], the inverse Green's function $d_{f s}^{-1}$ has the same form as in Eq. (32), where we should just permute the terms $X(N)$ and $Z(N)$.

\section{E. Transmission and reflection coefficients of a finite layered media embedded between two fluids}

Consider a structure made of solid-fluid layered media and embedded between two fluids characterized by their mass densities $\rho_{1}$ and $\rho_{2}$ and sound velocities $v_{1}$ and $v_{2}$ (see Fig. 2). Consider now an incident longitudinal wave launched in fluid 1 and polarized in the sagittal plane $\left(x_{1}, x_{3}\right)$ (Fig. 2). This incident wave takes the following form: 


$$
U_{i}\left(x_{3}\right)=A_{i} \frac{v_{1} k_{\|}}{\omega}\left(\begin{array}{c}
1 \\
\frac{i \alpha_{1}}{k_{\|}}
\end{array}\right) e^{-\alpha_{1} x_{3}},
$$

where $A_{i}$ is the amplitude of the incident field and $\alpha_{1}$ $=j \sqrt{\frac{\omega^{2}}{v_{1}^{2}}-k_{\|}^{2}}$. This yields reflected and transmitted waveswhich can be written, respectively, as

$$
u_{r}\left(x_{3}\right)=A_{r} \frac{v_{1} k_{\|}}{\omega}\left(\begin{array}{c}
1 \\
\frac{-i \alpha_{1}}{k_{\|}}
\end{array}\right) e^{\alpha_{1} x_{3}}
$$

and

$$
u_{t}\left(x_{3}\right)=A_{t} \frac{v_{2} k_{\|}}{\omega}\left(\begin{array}{c}
1 \\
\frac{i \alpha_{2}}{k_{\|}}
\end{array}\right) e^{-\alpha_{2}\left(x_{3}-L\right)},
$$

where $A_{r}$ and $A_{t}$ are the amplitudes of the reflected and transmitted waves, respectively. $\alpha_{2}=j \sqrt{\frac{\omega^{2}}{v_{2}^{2}}-k_{\|}^{2}}$ and $L$ is the total length of the multilayered structure.

The transmission coefficient through the finite system can be obtained from the third term in Eq. (7), namely,

$$
u_{t}\left(x_{3}\right)=G_{2}\left(x_{3}, L\right) G_{2}^{-1}(L, L) g(L, 0) G_{1}^{-1}(0,0) U_{i}(0) .
$$

From Eqs. (34), (36), and (37), one obtains

$$
A_{t}=A_{i} \frac{v_{1}}{v_{2}} \frac{2 \rho_{1} \omega^{2}}{\alpha_{2}} g(L, 0)
$$

where $g(L, 0)$ is the $x_{3} x_{3}$ component of the Green's function that relates the interfaces $L$ and 0 at both extremities of the finite structure. Therefore, the amplitude of the transmission coefficient can be derived from Eq. (41) as

$$
t=\frac{A_{t}}{A_{i}}=-\frac{v_{1} \alpha_{1}}{v_{2} \alpha_{2}} 2 F_{1} g(L, 0),
$$

where $F_{1}$ is defined as in Eq. (9): $F_{1}=-\frac{\rho_{1} \omega^{2}}{\alpha_{1}}$.

By the same way, the reflection coefficient is given by the second and third terms in Eq. (7), namely,

$$
\begin{aligned}
u_{r}\left(x_{3}\right)= & -G_{1}\left(x_{3}, 0\right) G_{1}^{-1}(0,0) U_{i}(0) \\
& +G_{1}\left(x_{3}, 0\right) G_{1}^{-1}(0,0) g(0,0) G_{1}^{-1}(0,0) U_{i}(0) .
\end{aligned}
$$

From Eqs. (34), (35), and (40), one obtains

$$
A_{r}=A_{i}\left[1-\frac{2 \rho_{1} \omega^{2}}{\alpha_{1}} g(0,0)\right],
$$

where $g(0,0)$ is the $x_{3} x_{3}$ component of the Green's function at the interface between fluid 1 and the multilayers. Therefore, the amplitude of the reflection coefficient can be derived from Eq. (41) as

$$
r=\frac{A_{r}}{A_{i}}=1+2 F_{1} g(0,0) .
$$

Equations (39) and (42) show that the calculation of transmission and reflection coefficients requires the knowledge of only the $x_{3} x_{3}$ component of the Green's function in the space of interfaces at the extremities of the whole system.

The reflection and transmission rates are given as follows:

$$
R=|r|^{2}
$$

and

$$
T=|t|^{2} \frac{\rho_{2} \alpha_{1}}{\rho_{1} \alpha_{2}} .
$$

The term $\frac{\rho_{2} \alpha_{1}}{\rho_{1} \alpha_{2}}$ is a correction term that ensures the conservation of sound power through such supposed lossless systems.

\section{F. Relation between the density of states and the group delay times}

Consider a finite layered structure inserted between two different semi-infinite fluids labeled 1 and 2 (Fig. 2). The inverse of the Green's function in the interface space $M$ is formed here by the two planes separating these three media $(\mathrm{M}=\{0, L\})$.

For each sagittal mode, the $x_{3} x_{3}$ component of the abovedefined $[g(M M)]^{-1}$ can be obtained from the surface $\left[g_{i}(M M)\right]^{-1}$ of these three media, namely,

$$
\left[g_{i}(0,0)\right]^{-1}=-F_{i}
$$

for the two semi-infinite fluids $i=1$ and 2 [Eq. (15)] and

$$
\left[g_{L}(M M)\right]^{-1}=\left(\begin{array}{ll}
A_{1} & B^{\prime} \\
B^{\prime} & A_{2}
\end{array}\right)
$$

for the layered media with free surfaces.

The detailed expressions for $A_{1}, A_{2}$, and $B^{\prime}$ are given in Sec. II D for finite solid-fluid SLs with different terminations. $A_{1}$ and $A_{2}$ are identical (different) for symmetrical (asymmetrical) structures [see Eqs. (27) and (32)]. The important point to notice is that these three quantities are purely real functions in a finite system; however, $F_{1}$ and $F_{2}$ are pure imaginary functions for the semi-infinite fluid media [Eq. $(9 \mathrm{c})]$. Therefore, $[g(M M)]^{-1}$ of the whole composite system can be obtained as follows: ${ }^{41}$

$$
[g(M M)]^{-1}=\left(\begin{array}{cc}
A_{1}-F_{1} & B^{\prime} \\
B^{\prime} & A_{2}-F_{2}
\end{array}\right) .
$$

From Eqs. (39) and (47), one obtains the transmission coefficient as follows:

$$
t=\frac{v_{1} \alpha_{1}}{v_{2} \alpha_{2}} 2 F_{1} B^{\prime}\{\operatorname{det}[g(M M)]\},
$$

where $\operatorname{det}[g(M M)]=\left[A_{1} A_{2}-B^{\prime 2}+F_{1} F_{2}-F_{1} A_{2}-F_{2} A_{1}\right]^{-1}$.

The reflection coefficient is given by Eqs. (42) and (47),

$$
r=\left[A_{1} A_{2}-B^{\prime 2}-F_{1} F_{2}+F_{1} A_{2}-F_{2} A_{1}\right] \operatorname{det}[g(M M)] .
$$

From Eqs. (48) and (49), one can obtain the phases $\theta_{T}$ and $\theta_{R}$ of the transmission and reflection coefficients. Of more interest are the derivatives of these phases with respect to the frequency that are indicative of the times needed by a wave 
packet to complete the transmission or reflection processes. These quantities, called group delay times (also referred to as the phase times in the literature ${ }^{44,45}$ ), are defined by

$$
\tau_{T}=\frac{d \theta_{T}}{d \omega}
$$

and

$$
\tau_{R}=\frac{d \theta_{R}}{d \omega} .
$$

From Eqs. (48) and (49), one can deduce that the transmission delay time can be written as

$$
\tau_{T}=\frac{d}{d \omega} \arg \{\operatorname{det}[g(M M)]\}+\pi \sum_{n} \operatorname{sgn}\left[\left.\frac{d B^{\prime}}{d \omega}\right|_{\omega=\omega_{n}}\right] \delta\left(\omega-\omega_{n}\right) .
$$

The reflection delay time $\tau_{R}$ can also be derived from Eqs. (49) as

$$
\begin{aligned}
\tau_{R}= & \frac{d}{d \omega} \arg \{\operatorname{det}[g(M M)]\} \\
& +\frac{d}{d \omega} \arg \left(A_{1} A_{2}-B^{\prime 2}-F_{1} F_{2}+F_{1} A_{2}-F_{2} A_{1}\right) .
\end{aligned}
$$

Let us now recall ${ }^{46}$ that the difference of the DOS between the present composite system and a reference system formed out of the same volumes of the semi-infinite fluids 1 and 2 and the finite structure can be obtained from

$$
\Delta n(\omega)=\frac{1}{\pi} \frac{d}{d \omega} \arg \{\operatorname{det}[g(M M)]\} .
$$

From Eqs. (51) and (53) one can deduce two cases:

(i) If the structure do not present transmission zeros (i.e., $\left.B^{\prime} \neq 0\right)$, then $\arg \left(B^{\prime}\right)=0$ and

$$
\tau_{T}=\pi \Delta n(\omega)
$$

(ii) If the transmission zeros occur at some frequencies, then the transmission coefficient changes sign, its phase exhibits a jump of $\pi$, and

$$
\tau_{T} \neq \pi \Delta n(\omega)
$$

Equations (52) and (53) show that $\tau_{R}$ is in general different from $\Delta n(\omega)$. However, if medium 2 is evanescent (i.e., $F_{2}$ is real and the incident wave is totally reflected). Then

$$
\tau_{R}=2 \pi \Delta n(\omega) \text {. }
$$

It is worth noticing that the calculation of the group delay time enables to deduce the group velocity in such structures using the relation ${ }^{47}$

$$
v_{g}=L / \tau,
$$

where $L$ represents the size of the structure.

\section{APPLICATION TO A FINITE SYMMETRIC SL EMBEDDED IN A FLUID}

\section{A. Band-gap structure and conditions for band and gap closing}

In order to illustrate the general results given before, we present here a simple application for sagittal acoustic waves in the special case of periodic solid plates immersed in the same fluid (for example, water). In this case, the transmission and reflection coefficients are given by Eqs. (48) and (49), namely,

$$
t_{N}=2 F \frac{B(N)}{A^{2}(N)-B^{2}(N)+F^{2}-2 F A(N)}
$$

and

$$
r_{N}=\frac{A^{2}(N)-B^{2}(N)-F^{2}}{A^{2}(N)-B^{2}(N)+F^{2}-2 F A(N)},
$$

where $A(N), B(N)$, and $F$ are given by Eqs. (28), (29), and (9), respectively.

In the particular case of one solid layer inserted in the fluid (i.e., $N=1$ ), one can show easily that $A(N)=A$ and $B(N)=B$ [Eqs. (28) and (29)], and $t_{N}$ and $r_{N}$ become, respectively,

$$
t_{1}=2 F \frac{B}{A^{2}-B^{2}+F^{2}-2 F A}
$$

and

$$
r_{1}=\frac{A^{2}-B^{2}-F^{2}}{A^{2}-B^{2}+F^{2}-2 F A} .
$$

Also, it is worth noticing that the numerator of $r_{N}$ [Eq. (59)] can be written after some algebraic calculation as

$$
A^{2}(N)-B^{2}(N)-F^{2}=\left[A^{2}-B^{2}-F^{2}\right]\left(\frac{t^{2 N}-1}{t^{2}}\right)\left(\frac{Y_{1}^{2}-Y_{2}^{2}}{\Delta}\right) \text {. }
$$

Equations (59) and (62) clearly show that the reflection zeros associated to a finite SL made of $N$ plates inserted periodically in a fluid are given either by

$$
A^{2}-B^{2}-F^{2}=0 \text {, }
$$

which coincides also with the reflection zeros associated to just one solid layer inserted in the fluid [Eq. (61)], or

$$
\sin (N k D)=0
$$

i.e.,

$$
k D=\frac{m \pi}{N}, \quad m=1,2, \ldots \ldots, N-1 .
$$

The third term in the right-hand side of Eq. (62) cannot vanish as $Y_{1} \neq \pm Y_{2}$.

The transmission zeros are given by $B(N)=0$ [Eq. (58)] or equivalently $B=0$ [see Eq. (29)]. This result shows that the transmission zeros of the whole structure coincide exactly with the transmission zeros of just one solid layer inserted in 


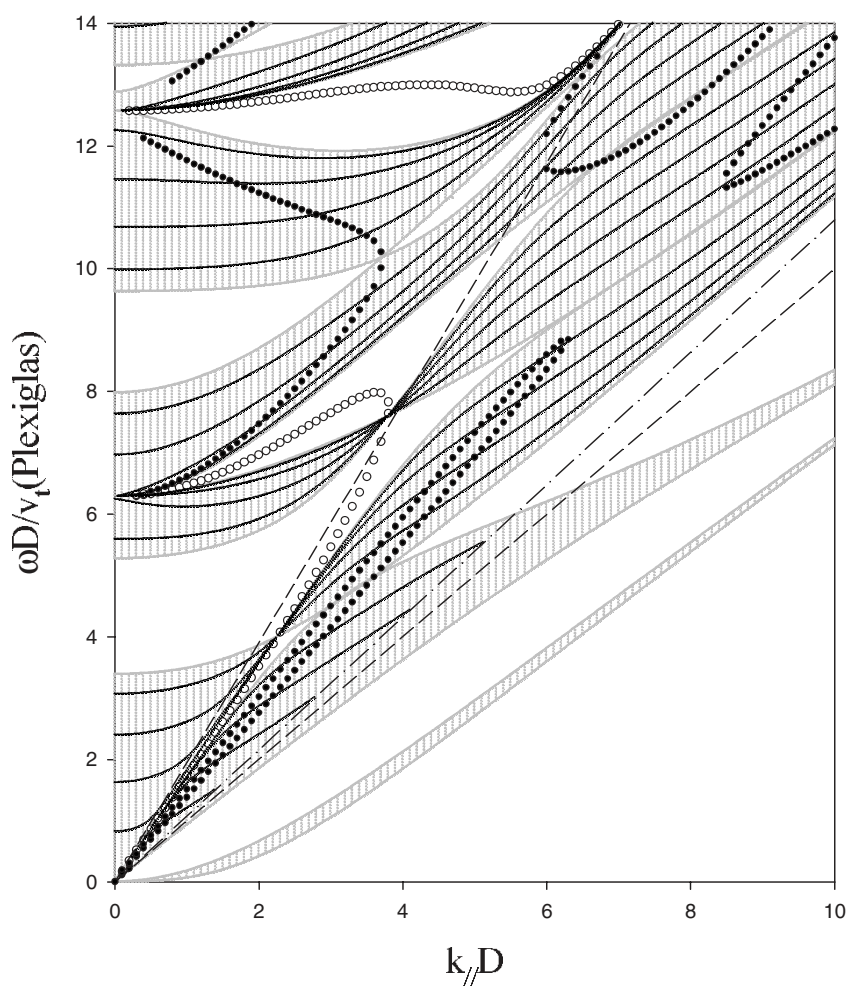

FIG. 3. Dispersion curves for a SL made of Plexiglas and water layers. The curves give $\Omega=\omega D / v_{t \text { (Plexiglas) }}$ as a function of $k_{\|} D$, where $\omega$ is the frequency, $k_{\|}$is the propagation vector parallel to the interface, $v_{t(\text { Plexiglas })}$ is the transverse speed of sound in Plexiglas, and $D$ is the period of the SL. The widths of fluid and solid layers are supposed equal: $d_{f}=d_{s}=D / 2$. The gray areas represent the bulk bands for an infinite SL. The thin solid lines and dotted curves show the positions of the reflection zeros (total transmission). Whereas, the open circles give the positions of the transmission zeros (total reflection). The dashed straight lines represent the transverse and longitudinal velocities of sound in Plexiglas. The dashed-dotted line represents the longitudinal velocity of sound in water.

the fluid [Eq. (60)]. In addition, the parameter $B$ characterizes only the solid layer [Eq. (11)]; therefore, the transmission zeros are independent on the choice of the fluid surrounding the solid layer, but depend only on the thickness and the elastic parameters of the solid.

Figure 3 gives the dispersion curves (gray areas) for an infinite SL made of Plexiglas and water layers. In this figure we plotted the reduced frequency $\Omega=\omega D / v_{t}$ (Plexiglas) as a function of the reduced wave vector $k_{\|} \mathrm{D}$. The thickness of solid and fluid layers are assumed to be equal $d_{s}=d_{f}=D / 2$ as in Refs. 5 and 6. Table I gives the numerical values of velocities of sound and mass densities of the materials used in this work. The dashed straight lines represent the transverse and longitudinal velocities of sound in Plexiglas, whereas the dashed-dotted line gives the longitudinal velocity of sound in water. The thin solid and dotted curves represent the dispersion curves obtained from the reflection zeros (total transmission) for a finite SL composed of $N=5$ Plexiglas layers inserted in water. The thin solid curves correspond to the $N$ -1 branches given by Eq. (64), whereas the dotted curves are given by Eq. (63). The open circles curves show the positions of the transmission zeros (total reflection). One can
TABLE I. Speed velocities of sound and mass densities of Plexiglas, aluminum, PVC, water, and mercury.

\begin{tabular}{lccc}
\hline \hline & $\begin{array}{c}\rho \\
\left(\mathrm{g} / \mathrm{cm}^{3}\right)\end{array}$ & $\begin{array}{c}v_{t} \\
\left(10^{5} \mathrm{~cm} / \mathrm{s}\right)\end{array}$ & $\begin{array}{c}v_{\ell} \\
(\mathrm{km} / \mathrm{s})\end{array}$ \\
\hline Plexiglas & 1200 & 1.38 & 2.7 \\
Aluminum & 2700 & 3.15 & 6.45 \\
PVC & 1200 & 1.03 & 2.3 \\
Water & 1000 & & 1.49 \\
Mercury & 13500 & & 1.45 \\
\hline \hline
\end{tabular}

notice a shrinking of the $N-1$ branches when they intercept the transmission zero branch around $\Omega=4.07, k_{\|} D=2.3$ and $\Omega=7.64, k_{\|} D=3.8$. This phenomenon reproduces for other values of the couple $\left(\Omega, k_{\|} \mathrm{D}\right)$ not shown here. This property of the shrinking of the modes is a characteristic of solid-fluid SLs and is without analog in their counterpart solid-solid SLs. ${ }^{7}$

Now, if we compare together the different branches associated to reflection and transmission zeros and the band-gap structure of the infinite Plexiglas-water SL, one can notice (Fig. 3) the following:

(i) As predicted, the thin solid and dotted curves corresponding to total transmission fall inside the allowed bands (gray areas), in particular, the positions of the closing of the gaps are given by the intersection of the limits of the band gaps and the dotted curves, i.e., we should have simultaneously

$$
\cos \left(k_{3} D\right)=\frac{A^{2}-B^{2}+F^{2}+2 A a}{B b}= \pm 1
$$

and

$$
A^{2}-B^{2}-F^{2}=0 .
$$

(ii) The open circle curves with total reflection (zero transmission) fall inside the forbidden bands, and the position of the closing of the bands should satisfy the two following conditions:

$$
\cos \left(k_{3} D\right)=\frac{A^{2}-B^{2}+F^{2}+2 A a}{B b}= \pm 1
$$

and

$$
B=0 .
$$

These particular crossings of the gaps give rise to a no dispersive curves (flat bands) for which the group velocity vanishes.

It is worth noticing that the transmission zeros (open circles) fall above a straight line, i.e., below a critical angle $\theta_{\mathrm{cr}}$. Indeed, a simple Taylor expansion of the function $B(\omega)$ in Eqs. (11) and (12) at the low-frequency limit (i.e., around $\omega \simeq 0$ and $k_{\|} \simeq 0$ ) gives

$$
\frac{\omega}{k_{\|}}=2 v_{t} \sqrt{1-\left(\frac{v_{t}}{v_{\ell}}\right)^{2}}=v_{\mathrm{cr}} .
$$



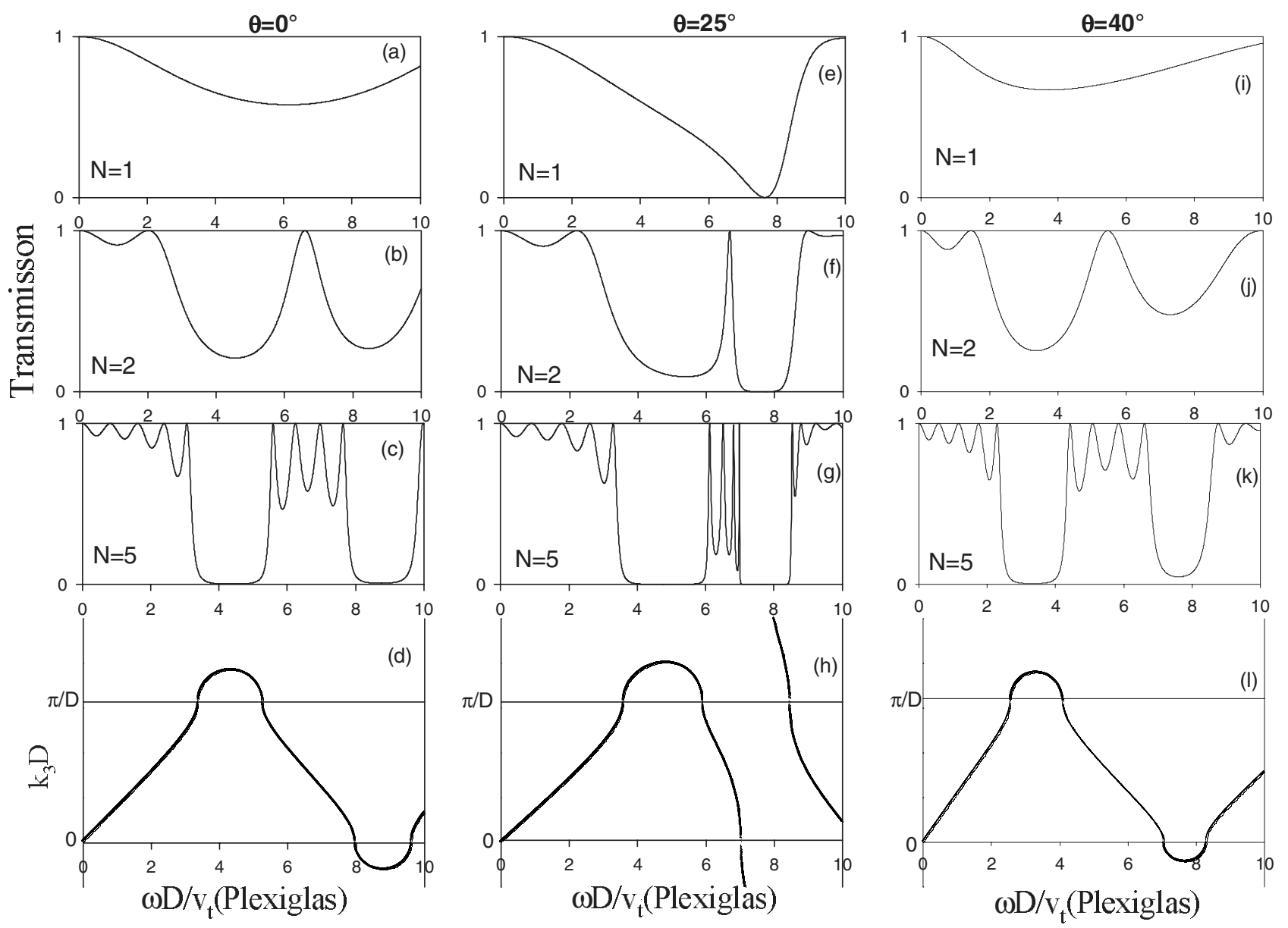

FIG. 4. Variation in the transmission coefficients as a function of the reduced frequency $\Omega$ for a finite SL composed of $N=1$ [(a), (e), and (i)], 2 [(b), (f), and (j)], and $5[(\mathrm{c}),(\mathrm{g})$, and (k)] Plexiglas layers immersed in water. The left, middle, and right panels correspond to incident angles: $\theta=0^{\circ}, 25^{\circ}$, and $40^{\circ}$, respectively. (d), (h), and (l) give the dispersion curves (i.e., $\Omega$ versus the Bloch wave vector $k_{3}$ ) inside the reduced Brillouin zone $0<k_{3}<\pi / D$. Outside this zone are represented the imaginary parts of $k_{3}$.

However, $k_{\|}$is related to the incident angle $\theta$ by the relation $k_{\|}=\frac{\omega}{v_{f}} \sin (\theta)$ (see Fig. 2). Thus, one obtains transmission zeros for wave velocities $v>v_{\text {cr }}$, or equivalently

$$
\theta<\theta_{\mathrm{cr}}=\arcsin \left(\frac{\frac{v_{f}}{v_{t}}}{2} \frac{1}{\sqrt{1-\left(\frac{v_{t}}{v_{\ell}}\right)^{2}}}\right)=39^{\circ} .
$$

However, let us mention that at normal incidence (i.e., $k_{\|}$ $=0$ or $\left.\theta=0^{\circ}\right), B(\omega)$ cannot vanish, and one obtains the wellknown dispersion relation ${ }^{35-37}$

$$
\cos \left(k_{3} D\right)=C_{\ell} C_{f}+\frac{1}{2}\left(\frac{Z_{\ell}}{Z_{f}}+\frac{Z_{f}}{Z_{\ell}}\right) S_{\ell} S_{f}
$$

where $Z_{\ell}=\rho_{s} v_{\ell}$ and $Z_{f}=\rho_{f} v_{f}$ are the acoustic impedances of longitudinal waves in solid and fluid layers, respectively. The above results show that the transmission zeros occur only for incidence angles $\theta$ such that $0^{\circ}<\theta<\theta_{\text {cr. }}$.

Figure 4 gives the variation in the transmission rates $T$ [Figs. 4(a)-4(c), 4(e)-4(g), and 4(i)-4(k)] as a function of the reduced frequency $\Omega$ for a finite SL composed of $N=1,2$, and 5 Plexiglas layers immersed in water. The left, middle, and right panels correspond to incident angles $\theta=0^{\circ}, 25^{\circ}$, and $40^{\circ}$, respectively. At the bottom of these panels we plotted the corresponding dispersion curves (i.e., $\Omega$ versus the Bloch wave vector $k_{3}$ ) [Figs. 4(d), 4(h), and 4(l)]. As predicted above, for $\theta=0^{\circ}$ (left panel) and $\theta>\theta_{c r}$ (right panel), the transmission exhibits dips at some frequency regions, which transform into gaps as far as $N$ increases. These gaps are due to the periodicity of the system (Bragg gaps) and coincide with the band-gap structure of the infinite SL shown in Figs. 4(d) and 4(l). For an incident angle $0^{\circ}<\theta<\theta_{\text {cr }}$ (middle panel), one can notice the existence of a transmission zero around $\Omega=7.64$ [Fig. 4(e)], which is due to the insertion of one Plexiglas layer $(N=1)$ in water. This transmission zero transforms to a large gap when $N$ increases. Besides this gap there exists a dip around $\Omega=5$ for $N=2$ [Fig. 4(f)], which also transforms to a gap when $N$ increases; this gap is due to the periodicity of the structure. The transmission gaps map the band-gap structure of the infinite SL [Fig. 4(h)], where one can notice that the imaginary part of the Bloch wave vector (responsible of the attenuation of the waves associated to defect modes) is finite in the Bragg gaps and tends to infinity inside the gaps due to the transmission 
zeros. These latter gaps can be used to localize strongly defect modes within the structure (see below).

From all the above results, one can conclude that for an incident angle $0^{\circ}<\theta<\theta_{\text {cr }}$ (middle panel) there exists two types of gaps: Bragg gaps, which are due to the periodicity of the structure, and gaps that are induced by the transmission zeros. However, at normal incidence $\left(\theta=0^{\circ}\right)$ (left panel) and for $\theta>\theta_{\text {cr }}$ (right panel) all the gaps are due to the periodicity of the system.

\section{B. Brewster acoustic angle}

Another interesting result that may be exhibited by solidfluid layered media is the possibility of existence of Brewster acoustic angles as for electromagnetic waves in dielectric media. ${ }^{2}$ The Brewster angle corresponds to an incident angle between two homogenous media for which there is no reflection. By analogy with transverse magnetic waves in $1 \mathrm{D}$ photonic crystals, the existence of such angles for transverse acoustic waves between two solids has been shown. ${ }^{48}$ This angle leads to the shrinking of the SL gaps to zero along a straight line whose slope is defined by the Brewster condition. The reflection zeros between solid and fluid media can be obtained by matching the Green's function of a semiinfinite solid [Eq. (15)] with that of a semi-infinite fluid [Eq. (16)], namely,

$$
\rho \frac{v_{t}^{4}}{\omega^{2} \alpha_{\ell}}\left(k_{\|}^{2}+\alpha_{t}^{2}\right)^{2}-4 \rho \frac{v_{t}^{4}}{\omega^{2}} \alpha_{t} k_{\|}^{2}=\rho_{f} \frac{\omega^{2}}{\alpha_{f}} .
$$

In the velocity region $v_{t}<v<v_{\ell}, \alpha_{\ell}$ is real (evanescent wave), whereas $\alpha_{t}$ and $\alpha_{f}$ are pure imaginary (propagative waves). Therefore, Eq. (72) is satisfied if

$$
\frac{v_{f}}{v_{t}}=\sqrt{\frac{2}{1+\frac{\rho_{f}^{2}}{\rho^{2}}}}
$$

and

$$
\omega=\sqrt{2} k_{\|} v_{t}
$$

or equivalently

$$
\theta_{B}=\arcsin \frac{v_{f}}{\sqrt{2} v_{t}},
$$

where $\theta_{B}$ is the Brewster angle. Through such angle, the incident longitudinal wave in the fluid enters completely the solid but converts to transverse wave. By using Snell's Law, Eq. (74) shows that the wave enters the solid at $45^{\circ}$ to the interface. Let us mention that a study of the Brewster acoustic angles at the fluid-solid interface in all the velocity regions has been performed some years ago by Sotiropoulos et al. ${ }^{49,50}$

Now, if a layered structure is made from such solid-fluid interfaces, then an incident wave will be totally transmitted, giving rise to the closing of the gaps along a straight line corresponding to Brewster angle. Such an angle is independent of the thickness of the layers in the SL, as well as on the longitudinal velocity of sound in the solid [see Eq. (74)]. In

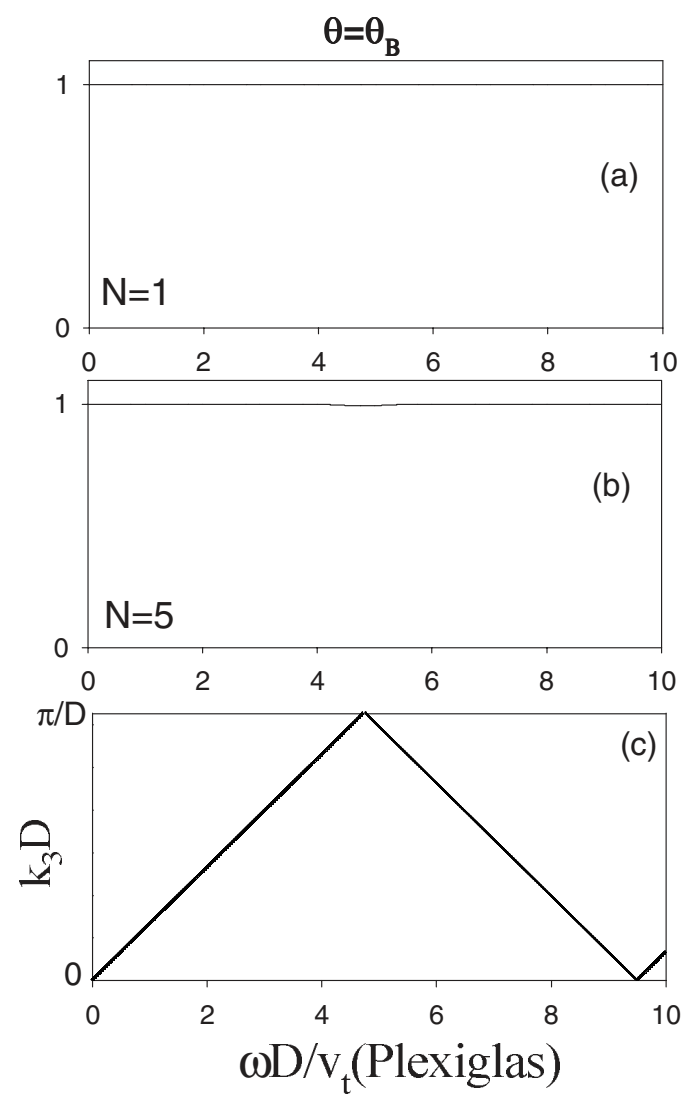

FIG. 5. Same as in Fig. 4 but for the Brewster angle: $\theta=\theta_{B}$ $=49^{\circ}$.

general, Eq. (73) is not easy to be satisfied by solid and fluid materials. However, in the case of Plexiglas-water structure considered here, Eq. (73) is almost satisfied and Eq. (74) gives $\theta_{B}=49.77^{\circ}$. Now, if we take an incident angle near to $\theta_{B}$ one obtains almost total transmission as it is shown in Figs. 5(a) and 5(b) corresponding, respectively, to $N=1$ and 5 Plexiglas layers immersed in water. The dispersion curves [Fig. 5(c)] show clearly the closing of the gaps at the center and edges of the reduced Brillouin zone for this incidence angle.

\section{Comparative study of the DOS and group delay times}

A comparative analysis of the transmission delay time and the density of states (DOS) is given in Fig. 6. The group delay times $\tau(\omega)$ [Figs. 6(a)-6(c)] and the variation in the density of states $\Delta n(\omega)$ [Figs. 6(d)-6(f)] are plotted as functions of the reduced frequency $\Omega$ for a finite SL composed of $N=5$ Plexiglas layers immersed in water and for three incident angles: $\theta=0^{\circ}\left[\right.$ Figs. 6(a) and 6(d)], $\theta=30^{\circ}$ [Figs. 6(b) and $6(\mathrm{e})]$, and $\theta=60^{\circ}$ [Figs. 6(c) and 6(f)]. The group delay time gives information on the time spent by the phonon inside the structure before its transmission, while the DOS gives the weight of the modes. In Fig. 6, the DOS and the delay time are strongly reduced in the band-gap regions. As predicted by the analytical results in Sec. II F, the delay time may give rise to delta functions around the transmission zeros as it is shown in Fig. 6(b) around $\Omega \simeq 8$ for $0^{\circ}<\theta$ 

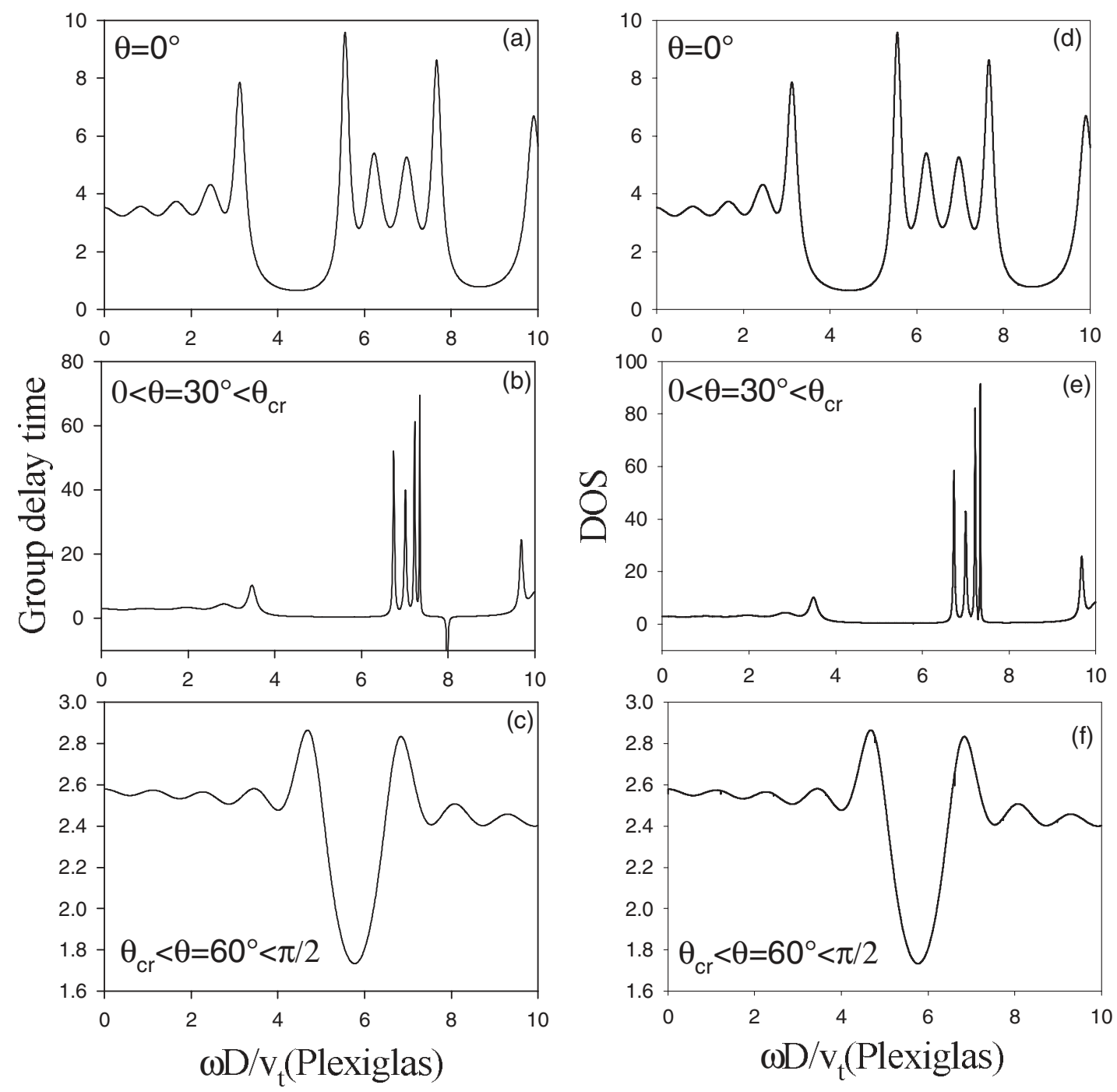

FIG. 6. Transmission delay time (left panel) and density of states (DOS) (right panel) [(in units of $D / v_{t}($ Plexiglas)) $]$ as a function of the reduced frequency $\Omega$ for a finite SL composed of $N=5$ Plexiglas layers immersed in water and for three incident angles: $\theta=0^{\circ}[(\mathrm{a})$ and (d)], $\theta=30^{\circ}[(\mathrm{b})$ and (e) $]$, and $\theta=60^{\circ}[(\mathrm{c})$ and (f)].

$<\theta_{\text {cr. }}$ This delta function, which does not exist in the DOS [Fig. 6(e)], has been enlarged by adding a small imaginary part to the pulsation $\omega$, which plays the role of absorption in the system. Such negative delta peaks have been shown experimentally in simple photonic ${ }^{51}$ and phononic ${ }^{52}$ loop waveguides, giving rise to the so-called superluminal or negative group velocity [Eq. (57)]. Because of the nonexistence of transmission zeros, solid-solid layered media do not exhibit such negative delay times or negative group velocities. Figures 6(a)-6(f) clearly show, in accordance with Eqs. (51) and (53), that except the frequencies lying around the transmission zeros, the DOS and the group delay time exhibit exactly the same behavior.

\section{GENERAL RULE ABOUT CONFINED AND SURFACE MODES IN A FINITE ASYMMETRIC SUPERLATTICE}

Recently ${ }^{40}$ we have demonstrated that the creation of two semi-infinite SLs from the cleavage of an infinite solid-fluid
SL gives rise to one surface mode by gap for any value of the wave vector $k_{\|}$. This mode belongs to one or the other of the two complementary SLs. In this paper, we give a generalization of these results to a finite-size SL made of $N$ solid-fluid cells [Figs. 1(c) and 1(d)] with both extremities in contact with vacuum. The expression giving the eigenmodes of such a structure is given by Eqs. (6), (32), and (33) and can be written in the following form:

$$
\left[t-\frac{a^{2}-b^{2}+A a}{B b}\right]\left[\frac{1}{t}-\frac{a^{2}-b^{2}+A a}{B b}\right]\left(1-t^{2 N}\right)=0 .
$$

This expression shows that there are two types of eigenmodes in this kind of finite structure:

(i) If the wave vector $k_{3}$ is real, which corresponds to an allowed band, then the eigenmodes of the finite SL are given by the third term in Eq. (75), namely, 


$$
\sin (N k D)=0
$$

which gives

$$
k D=\frac{m \pi}{N}, \quad m=1,2, \ldots \ldots, N-1,
$$

whereas the first and second terms in Eq. (75) cannot vanish in the bulk bands as $t=e^{j k_{3} D}$ is complex and $a, b, A$, and $B$ are real.

(ii) If the wave vector $k_{3}$ is imaginary (modulo $\pi$ ), which corresponds to a forbidden band, then the eigenmodes are given by the two first terms of Eq. (75), namely,

$$
t=\frac{a^{2}-b^{2}+A a}{B b}
$$

and

$$
\frac{1}{t}=\frac{a^{2}-b^{2}+A a}{B b} .
$$

These two expressions give the localized modes associated to the two surfaces surrounding the structure. The third term in Eq. (75) cannot vanish inside the gap since $t$ should satisfy the condition

$$
|t|<1
$$

to ensure the decaying of surface modes from the surface.

In addition, we remark that if $N \rightarrow \infty$ the term $t^{2 N}$ vanishes and, therefore, the two expressions [Eqs. (78) and (79)] give the surface modes for two semi-infinite SLs obtained from the cleavage of the infinite SL between the solid and fluid layers. ${ }^{40}$ Equation (75) clearly shows that the surface modes are independent of the number $N$ of cells in the finite system.

Equations (78) and (79) can be written in a unique explicit form by replacing them in Eq. (18) and factorizing by the factor $\frac{1}{B b}$, one obtains

$$
a\left(B^{2}-A^{2}\right)-A\left(a^{2}-b^{2}\right)=0 .
$$

Therefore, the surface modes associated to one surface are given by Eq. (81), together with the condition $\left|\frac{a B}{A b}\right|>1$ [Eqs. (78) and (80)], whereas the surface modes of the other surface are given by Eq. (81) but with the condition $\left|\frac{a B}{A b}\right|<1$ [Eqs. (79) and (80)]. This result shows that if a surface mode appears on one surface of the finite SL, it does not appear on the other surface. Equation (81) with the supplementary condition are similar to those given in Ref. 40 for semi-infinite SLs. The above results clearly show that a finite SL made of $N$ solid-fluid layers exhibits $N-1$ modes in each allowed band and one additional mode by gap induced by one of the two surfaces surrounding the structure. These results generalize our previous findings ${ }^{40}$ on semi-infinite solid-fluid SLs.

An example of the dispersion curves is given in Fig. 7 for a SL composed of $N=4$ Plexiglas-water cells. The other parameters are the same as in Fig. 3. One can notice the existence of $N-1=3$ modes in each band; these modes correspond to confined modes (stationary waves) and one surface branch in each gap induced by one or the other of the two surfaces ending the structure. The open circles and triangles correspond to surface modes induced by fluid and solid layer

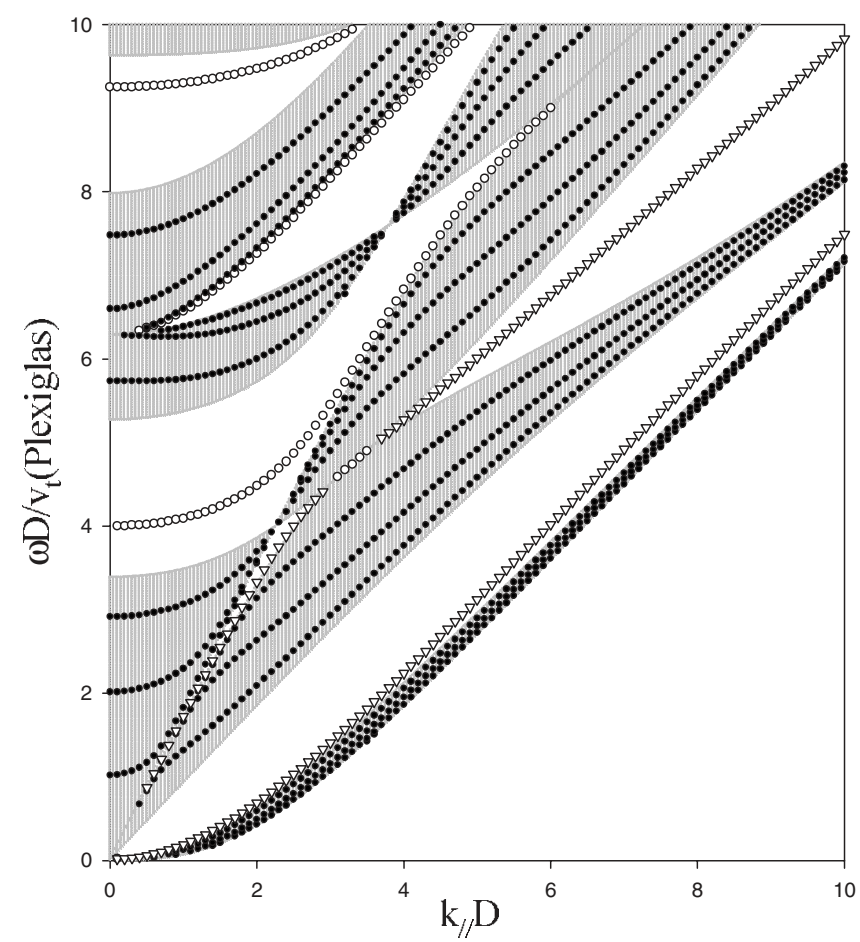

FIG. 7. Dispersion curves for a finite SL made of $N=4$ Plexiglas-water bilayers with free surfaces. The dotted curves represent $N-1=3$ confined modes in the finite SL. These modes fall inside the allowed bands of the SL. The open circles (open triangles) correspond to localized modes induced by the surface of the SL terminated by the fluid layer (solid layer). These modes fall inside the band gaps. The gray areas represent the bulk bands for the infinite SL.

terminations, respectively. As mentioned above, these modes coincide exactly with the surface modes of two complementary SLs obtained from the cleavage of an infinite SL between the solid and fluid layers. ${ }^{40}$ When $\mathrm{N}$ increases, the number of branches in each band increases, whereas the surface branches fall at the same frequencies.

The detection of such surface acoustic modes in a solidfluid SL with a fluid or a solid at the surface can be achieved by means of the reflection coefficient. Indeed, an incident wave launched from a semi-infinite fluid in contact with a finite SL terminated by a free surface will be totally reflected back. Therefore, the amplitude of the reflected wave is unity, and only its phase or equivalently the delay time may give information on the surface modes induced by the surface layer. ${ }^{6,7}$ Indeed, the surface modes appear as well-defined peaks in the delay time, which is equivalent to the density of states, as it was demonstrated in Eq. (56). Figure 8 gives the bulk and surface modes of a finite SL terminated by water. These modes are obtained from the maxima of the delay time, as it is illustrated in the inset for $k_{\|} D=1$. One can notice that the bulk modes (dots), as well as the surface modes (open circles), appear as well-defined peaks in the delay time, which represents the time needed for a phonon to accomplish the reflection process. The modes in Fig. 8 are resonant (or leaky) modes; the corresponding frequencies underwent a small shift in comparison with those in Fig. 7 because of their coupling with water radiation modes. In par- 


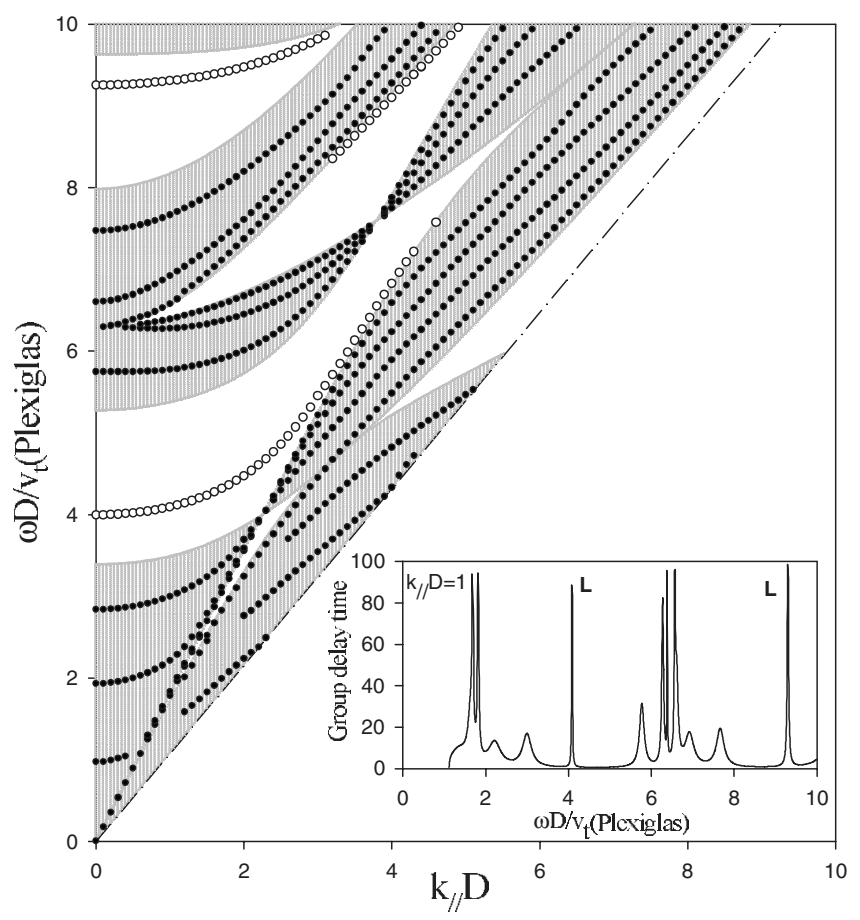

FIG. 8. Dispersion curves for a finite SL made of $N=4$ Plexiglas-water bilayers. The Plexiglas layer at one end is in contact with a semi-infinite fluid made of water, whereas the other surface is kept free of stress. The open circles represent the surface modes induced by the fluid layer at the free surface. These modes fall inside the band gaps. The dots represent the confined modes lying inside the allowed bands. All these modes are obtained from the maxima of the reflection delay time as it is illustrated in the inset of Fig. 8 (a) for $k_{\|} D=1$. $L$ denotes the surface modes induced by the water layer at the surface. The dashed-dotted line indicates the water sound line.

ticular, the surface modes in Fig. 8 (open circles) are almost the same as those in Fig. 7. Figure 9 gives the same information as in Fig. 8 but when the SL is terminated by the Plexiglas layer. Here also the bulk modes (dots) underwent a small shift in comparison with those in Fig. 7, but the surface modes (open triangles) are very close to those in Fig. 7.

It is worth to mention that the above-mentioned rule on confined and surface modes has been obtained recently by Ren et $a l .{ }^{53}$ for pure transverse elastic waves in solid-solid SLs. The same rule has been confirmed theoretically and experimentally ${ }^{54}$ by the authors for electromagnetic waves in quasi-1D structures made of coaxial cables.

\section{OMNIDIRECTIONAL ACOUSTIC BAND GAP IN SOLID-FLUID LAYERED MEDIA}

Some years ago, ${ }^{22}$ we have shown that $1 \mathrm{D}$ solid-solid layered structures can exhibit the property of omnidirectional reflection for acoustic waves. In the frequency range of the omnidirectional reflection, the structure will behave analogously to the case of $2 \mathrm{D}$ and $3 \mathrm{D}$ phononic crystals, i.e., it reflects any acoustic wave independent of its polarization and incidence angle. These results have been confirmed experimentally some years later ${ }^{23}$ by means of ultrasonic tech-

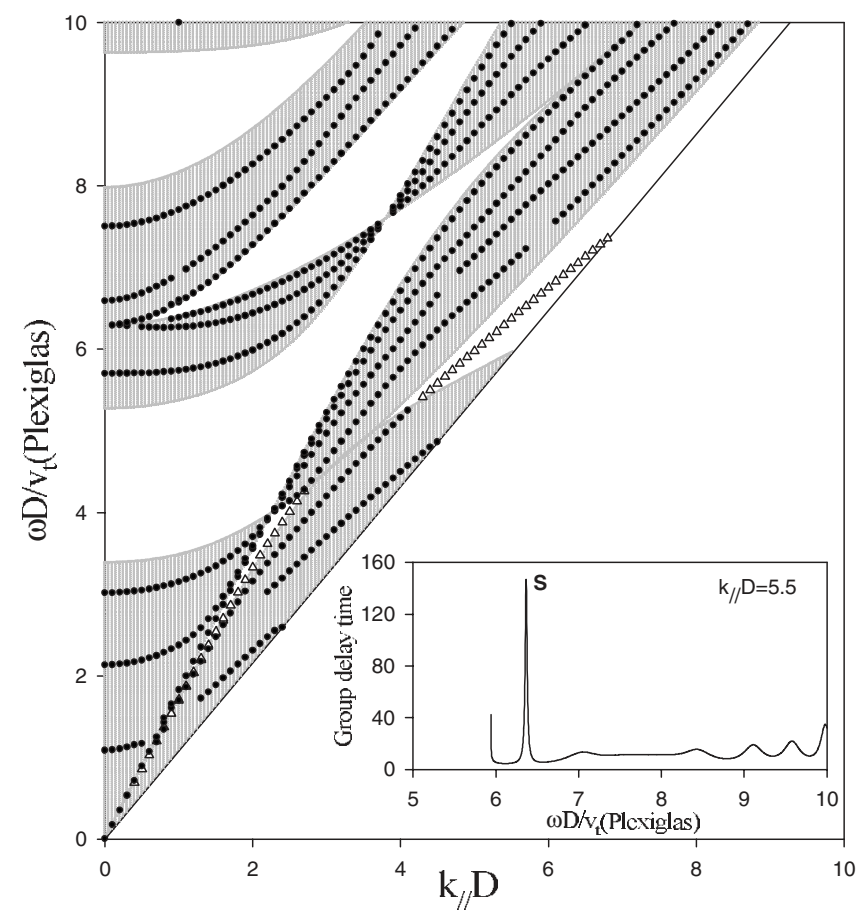

FIG. 9. The same as in Fig. 8, but for a SL terminated by a Plexiglas layer at the free surface. The triangles represent the surface modes induced by this layer. The inset gives an example of the reflection delay time for $k_{\|} D=5.5$. $S$ denotes the peak of the surface mode in the delay time.

niques. The object of this section is to examine the condition for the existence and behavior of omnidirectional band gaps in finite solid-fluid layered media. More precisely, the transmission spectra are calculated and analyzed in relation with the dispersion curves of the modes associated with the finite structure embedded between two semi-infinite fluids. When a maximum threshold for transmittance is imposed, we investigate the contributions of the different modes induced by the finite structure to the transmission spectra, thus, revealing the limitations on the existence of an absolute band gap.

Let us first come back to the band-gap structure given in Fig. 3. One can notice that the band-gap structure of the infinite Plexiglas-water SL does not display any absolute gap, this means a gap existing for every value of the wave vector $k_{\|}$. Figure 10(a) reproduces the results given in Fig. 3 for a finite Plexiglas-water SL made of $N=8$ cells. The discrete modes are obtained from the maxima of the transmission rate that exceeds a threshold fixed to $10^{-3}$. One can notice that any wave launched from water will display a partial gap for an incident angle $0^{\circ}<\theta<35^{\circ}$ in the frequency region $4.015<\Omega<5.105$ indicated by horizontal lines. However, waves with incident angles $35^{\circ}<\theta<90^{\circ}$ will be totally transmitted through the discrete modes of the $\mathrm{SL}$ as it was discussed in Sec. III A. These results remain valid for any incident liquid medium as, in general, the velocities of sound in most liquids are of the same order or less than water. In order to overcome this limitation or at least facilitate the existence of an omnidirectional gap, we proposed, like in our previous works in solid-solid SLs, ${ }^{22}$ two solutions. The first one consists to clad the SL on one side by 

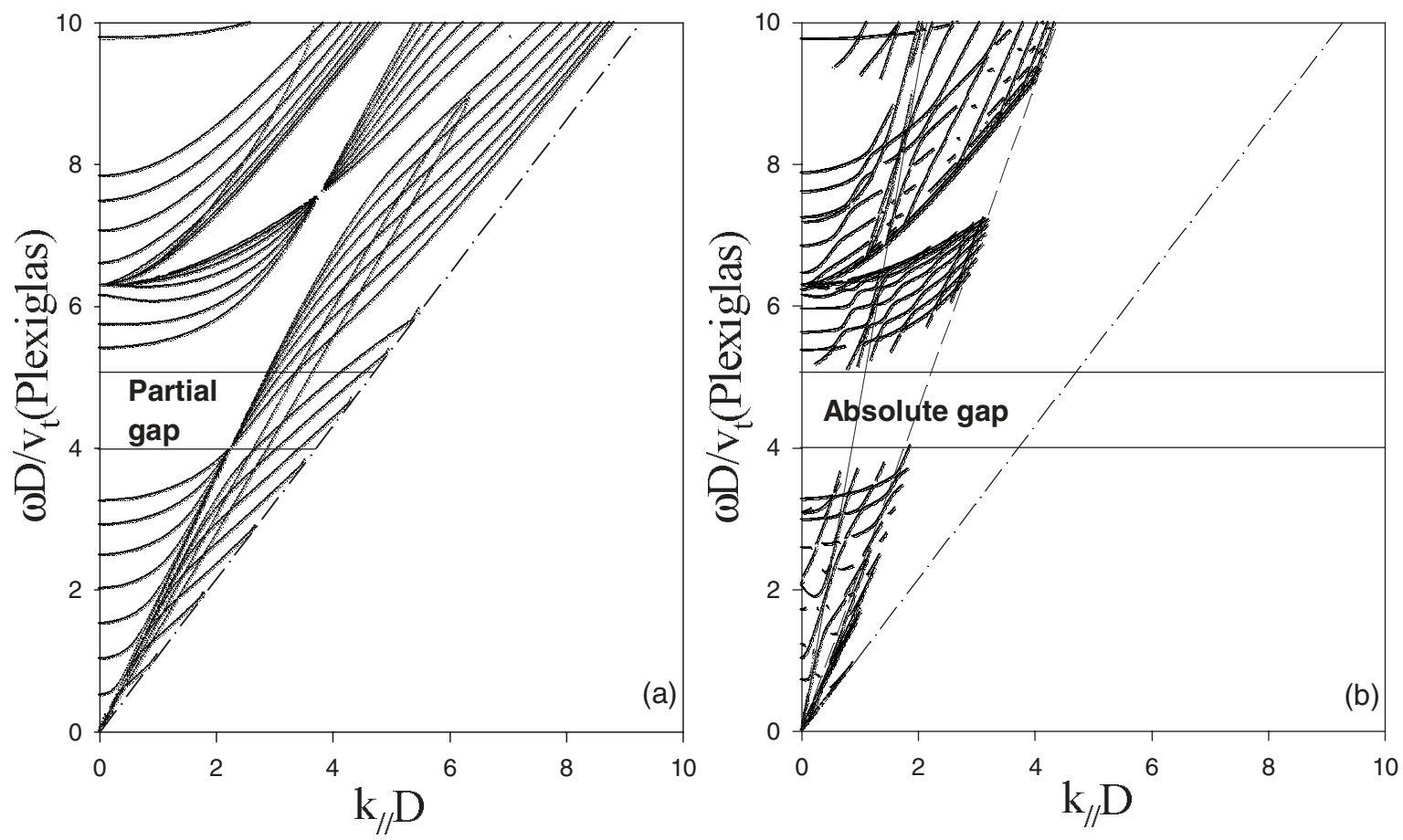

FIG. 10. (a) Dispersion curves of a finite SL composed of $N=8$ Plexiglas layers immersed in water. The discrete modes correspond to the frequencies obtained from the maxima of the transmission rate that exceeds a threshold of $10^{-3}$. (b) The same as (a) but here the SL is cladded with an Al layer of thickness $d_{0}=7 D$ on one side.

a buffer layer of high acoustic velocities, which can act as a barrier for the propagation of phonons. The second solution consists to associate in tandem two SLs in such a way that their band structures do not overlap. The calculation procedure used to deduce the expressions of transmission coefficients for a SL with a defect layer, as well as for the association of two SLs, will be developed later in Sec. VI.

\section{A. Cladded-superlattice structure}

Figure 10(b) gives the discrete modes associated to the cladded-SL structure, i.e., the frequency domains in which the transmission rate exceeds a threshold of $10^{-3}$. In this example the clad layer is made of $\mathrm{Al}$ with transverse and longitudinal velocities of sound (dashed and straight lines) higher than the SL bulk modes lying in the frequency region $4.015<\Omega<5.105$ [Fig. 10(b)]. The thickness of the Al layer is $d_{0}=7 D$ and the SL contains $N=8$ cells of Plexiglas-water. By combining these two systems, the allowed modes of the $\mathrm{SL}$ and the guided modes induced by the Al clad layer above its velocities of sound do not overlap over the frequency range of the omnidirectional gap. This means that each system acts as a barrier for phonons of the other system. In such a way, one obtains an omnidirectional band gap indicated by the two horizontal lines in Fig. 10(b) in the frequency region $4.015<\Omega<5.105$. By comparing Figs. 10(a) and 10(b), one can notice clearly that the presence of the clad layer has two opposite effects. It decreases the transmittance in some frequency domains (essentially below the sound line defined by the transverse velocity of sound in the clad), but also introduces new modes that can contribute themselves to transmission. The transmission by the latter modes is prevented by the SL when the corresponding branches fall inside the minigaps. In the allowed frequency regions belonging to both the SL and the clad layer, one can notice an interaction and an anticrossing of the modes associated to these two systems.

To give a better insight into the behavior of the transmission coefficient, we present in Fig. 11 the transmission rates through the cladded-SL system for three reduced wave vectors: $k_{\|} D=0$ [Fig. 11(a)], 1 [Fig. 11(b)], and 3 [Fig. 11(c)]. One can see clearly the common forbidden region in the transmission spectra indicated by the two vertical lines, showing the mirror effect played by the layered structure in the frequency region $4.015<\Omega<5.105$. For the sake of comparison, we have also given in Figs. 11(d)-11(f) the DOS (or equivalently the delay time). One can notice that the Al clad layer induces guided modes which appear as peaks in the DOS. These modes do not contribute to transmission when they fall inside the minigap of the SL as it is clearly shown for the modes lying in the frequency region 4.015 $<\Omega<5.105$ in Figs. 9(d)-9(f) in comparison to Figs. 9(a)-9(c), respectively.

The existence and behavior of the omnidirectional reflection depends on the geometrical parameters involved in the structure, namely, the thickness $d_{0}$ of the Al layer and the number $N$ of unit cells in the SL. In Fig. 12(a), we present the variation in the gap width as a function of $d_{0}$ for a finite $\mathrm{SL}$ made of $N=8$ cells. The omnidirectional gap widens with increasing the thickness of $\mathrm{Al}$ and reaches a constant width for $d_{0}>3 D$. Similarly, if the thickness of the Al layer is fixed to $d_{0}=4 D$, then a finite SL made of at least $N=7$ cells is required to reach a large omnidirectional gap [see Fig. 12(b)]. 

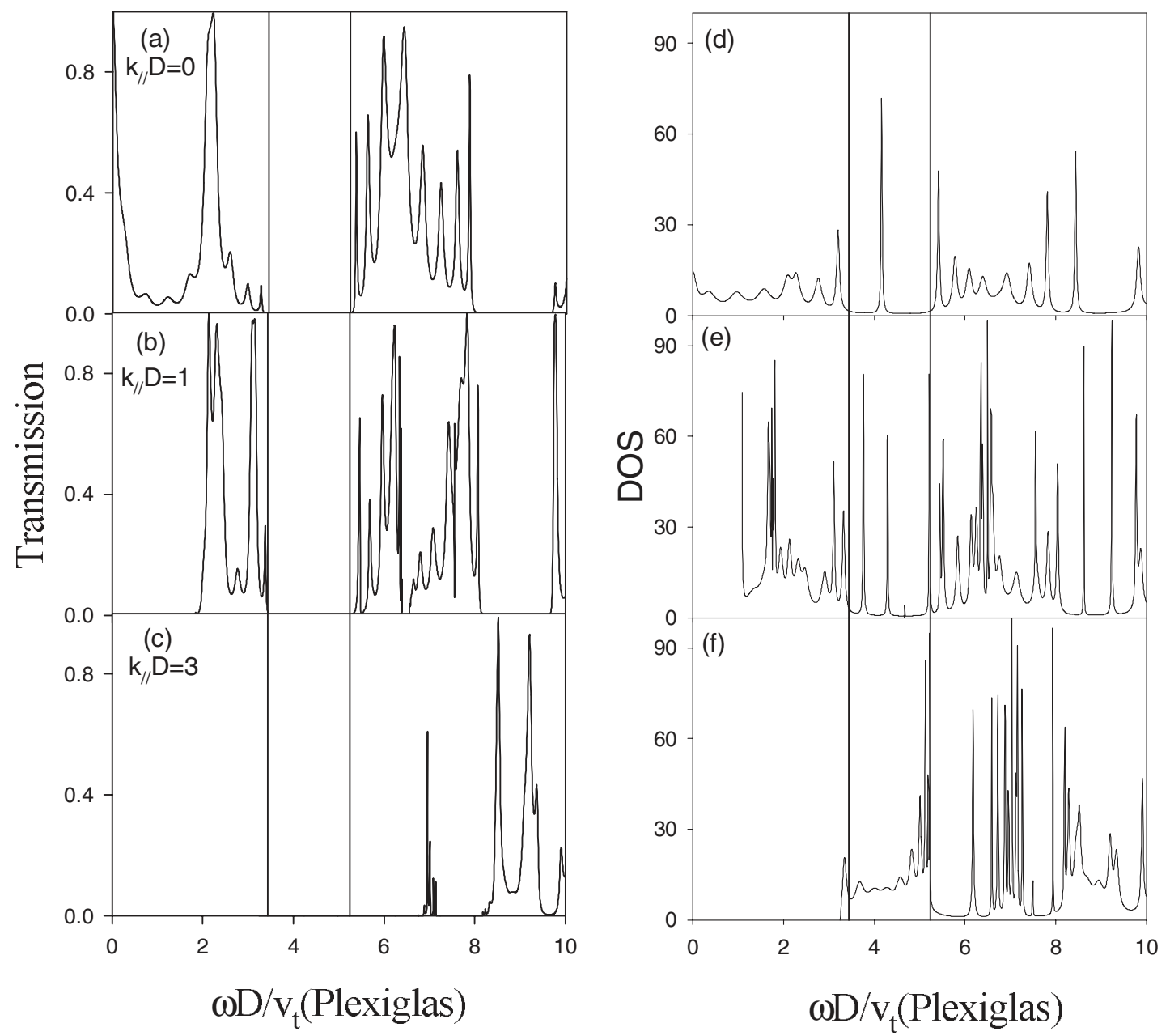

FIG. 11. Transmission rate (left panel) and density of states (DOS) (right panel) as a function of the reduced frequency $\Omega$ for the finite SL depicted in Fig. 10(b) and for three values of $k_{\|} \mathrm{D}: k_{\|} D=0$ (a) and (d), $k_{\|} D=1$ (b) and (e), and $k_{\|} D=2$ (c) and (f).

\section{B. Coupled multilayer structure}

The second solution that enables us to perform omnidirectional transmission gaps consists of considering a combination of two SLs chosen in such a way that the superposition of their band structures displays an absolute band gap. This means that the minibands of one SL overlap with the minigaps of the other SL, and vice versa in some frequency range. An example showing this property is given in Fig. 13(a) for a combination of the band structures of two SLs made of Plexiglas-Hg and PVC-Hg. The periods of the two SLs as well as the thickness of the corresponding layers are supposed to be equal: $d($ Plexiglas $)=d(\mathrm{PVC})=d(\mathrm{Hg})=D / 2$. The band structures of Plexiglas-Hg and PVC-Hg SLs are indicted by black and gray areas, respectively. The superposition of these two types of bands clearly displays two broad acoustic gaps in the frequency ranges $2.72<\Omega<4.94$ and $5.14<\Omega<5.96$. One can expect that in these frequency domains, an incident wave launched from any semi-infinite fluid will be totally reflected. In practice the two coupled SL structure is of finite width, and one can only impose a maximum threshold on the transmission coefficient $\left(T>10^{-3}\right)$.

An example is sketched in Fig. 13(b), where we have considered two finite SLs made of $N_{1}=4$ layers of Plexiglas and $N_{2}=4$ layers of PVC immersed in Hg. The two omnidirectional band gaps fall in the frequency ranges $2.72<\Omega$ $<4.94$ and $5.14<\Omega<5.96$ and practically coincide with acoustic band gap of Fig. 13(a). Similarly to the cladded-SL structure discussed above, the bulk modes of each of the two SLs may give rise to well-defined peaks in the DOS within the omnidirectional gaps (not shown here). However, these modes do not contribute to the transmission spectra. Obviously, the width and the position of the omnidirectional gaps depend upon the relative widths of the layers in each SL but also upon the numbers $N_{1}$ and $N_{2}$ of cells in each SL. Let us mention that a partial gap obtained from the association of two solid-fluid SLs has been shown theoretically and experimentally ${ }^{37}$ for normal incidence.

\section{SELECTIVE TRANSMISSION}

In this section we shall discuss the possibility of acoustic waves filtering through the band gap of solid-fluid layered media. This selective transmission can be realized either by inserting a defect layer within the finite SL or through the modes induced by the interface between the SL and an homogeneous fluid medium. 


\section{A. Transmission through resonant cavity modes}

The cavity modes can be created in the solid-fluid SL by replacing for example a fluid layer of width $d_{f}$ in the cell $(n=p)$ by a different fluid of width $d_{0}$ and characterized by the density $\rho_{0}$ and the sound velocity $v_{0}$. Consider a solidfluid SL with a finite number $N$ of cells and containing a defect fluid in the cell $p(1<p \leq N)$, the whole structure is inserted between two fluids characterized by their density $\rho_{s}$ and sound velocity $v_{s}$ [Fig. 1(e)]. The transmission coeffi- cient through the system described above can be obtained in the same way as for the structure without defect (Sec. III A). It consists in coupling two finite SLs made of $p$ and $N-p$ cells by a fluid layer and inserting the whole system between two fluid media [Fig. 1(e)]. The inverse of the Green's function in the space of interfaces $M=\left\{\left(1, f, \frac{d_{f}}{2}\right),(P+1, f\right.$, $\left.\left.-\frac{d_{f}}{2}\right),\left(P+1, f, \frac{d_{f}}{2}\right),\left(N+1, f,-\frac{d_{f}}{2}\right)\right\}$ of the whole system is given by a superposition of the Green's functions matrices associated to the different media constituting the system, namely,

$$
g^{-1}(M M)=\left(\begin{array}{cccc}
A(P)-F_{s} & B(P) & 0 & 0 \\
B(P) & A(P)+a_{0} & b_{0} & 0 \\
0 & b_{0} & A(N-P)+a_{0} & B(N-P) \\
0 & 0 & B(N-P) & A(N-P)-F_{s}
\end{array}\right),
$$

where $A(P), B(P), A(N-P)$, and $B(N-P)$ are defined by Eqs. (28)-(30) for $N=P$ and $N=N-P, a_{0}$ and $b_{0}$ are given by Eq. (9c) for the fluid labeled " 0 ," and $F_{s}$ is the same as in Eq. $(9 \mathrm{c})$ for the fluid labeled "s."

From Eqs. (82) and (39), one can deduce the transmission coefficient as follows:

$$
t=\frac{2 F_{s} b_{0} B(P) B(N-P)}{\Psi(P) \Psi(N-P)-b_{0}^{2}\left[A(P)-F_{s}\right]\left[A(N-P)-F_{s}\right]},
$$

where

$$
\Psi(l)=B^{2}(l)-[A(l)-F s]\left[A(l)+a_{0}\right]
$$

for $l=P, N-P$.

It is well known that the introduction of a defect layer (cavity) in a periodic structure can give rise to defect modes inside the band gaps. ${ }^{14-18,36}$ These modes appear as welldefined peaks in the DOS; however, their contribution to the transmission rate depends strongly on the position of these defects inside the structure. Indeed, as it was shown above, a defect layer placed at the contact between the SL and the substrate (clad layer) induces guided modes in the band gap of the SL but without contributing to the transmission. However, the transmission through these modes can be significantly enhanced if the cavity layer is placed at the middle of the structure. ${ }^{17,27}$ In general, a periodic structure made of $N$ cells $(N>2)$ is needed to create a transmission gap in which a defect mode is then introduced for filtering. In this subsection, we are interested to show that contrary to solid-solid SLs, it is possible to achieve large gaps, as well as sharp resonances inside these gaps with a solid-fluid structure as small as solid-fluid-solid sandwich triple layers. This property is associated with the existence of zeros of transmission.

Figure 14(a) gives the transmission rate as a function of the reduced frequency $\Omega$ for a finite Plexiglas-water SL composed of $N=2$ (solid curves) and $N=4$ (dotted curves) cells and for an incidence angle $\theta=35^{\circ}$. The fluid and solid layers have the same width $d_{f}=d_{s}=D / 2$. One can notice that the transmission rate exhibits a large dip in the frequency region $4<\Omega<8$ around the transmission zero indicated by an open circle on the abscissa. This transmission gap maps the band gap of the infinite system indicated by solid circles on the abscissa. As it was discussed above, the transmission gap becomes well defined as far as $N$ increases. Now, if a fluid cavity layer of thickness $d_{0}=D$ is inserted in the middle of the structure, then a resonance with total transmission can be introduced in the gap [Fig. 14(b)]. This resonance falls at almost the same frequency and its width decreases when $N$ increases. Let us mention that the structure depicted in Figs. 14(a) and 14(b) with $N=2$ consists on a sandwich system made of two Plexiglas layers separated by a water layer. Therefore, such a small-size structure clearly show the possibility of obtaining a large gap and a sharp resonance inside the gap by just tailoring the width of these three layered media. This property is specific to solid-fluid structures and is without analog for their counterpart solid-solid systems, where at least a number $N>2$ of layers is needed to achieve well-defined gaps and cavity modes. In what follows, we shall focus on the simple case of sandwich system (i.e., $N$ $=2$ ).

An important point to notice in Fig. 14(b) is the shape of the resonance lying in the vicinity of the transmission zero. Such a resonance is called Fano resonance. ${ }^{55}$ The origin and the asymmetry Fano profile of this resonance were explained as a result of the interference between the discrete resonance and the smooth continuum background in which the former is embedded. The existence of such resonances in 2D and 3D phononic crystals, the so-called locally resonant band-gap materials, ${ }^{56}$ has been shown recently. ${ }^{57}$ Some analytical models have been proposed to explain the origin and the behavior of these resonances. ${ }^{57}$ In the case of $1 \mathrm{D}$ model proposed here, the Fano resonance in Fig. 14(b) is just an internal resonance induced by the discreet modes of the fluid layer when these modes fall at the vicinity of the transmission 

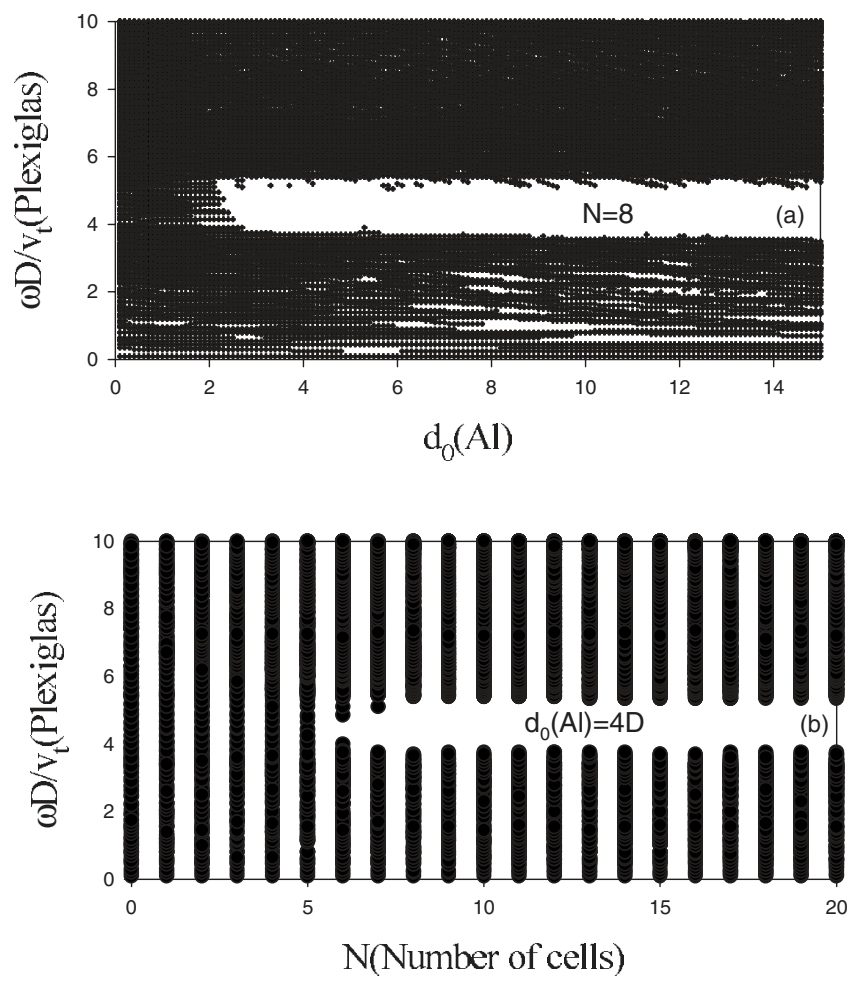

FIG. 12. (a) Variation in the omnidirectional gap width as a function of the thickness $d_{0}$ of the Al clad layer for a finite SL made of $N=8$ cells. (b) Variation in the omnidirectional gap width as a function of the number $N$ of unit cells in the finite SL for a fixed thickness of the Al layer such that $d_{0}=4 D$.

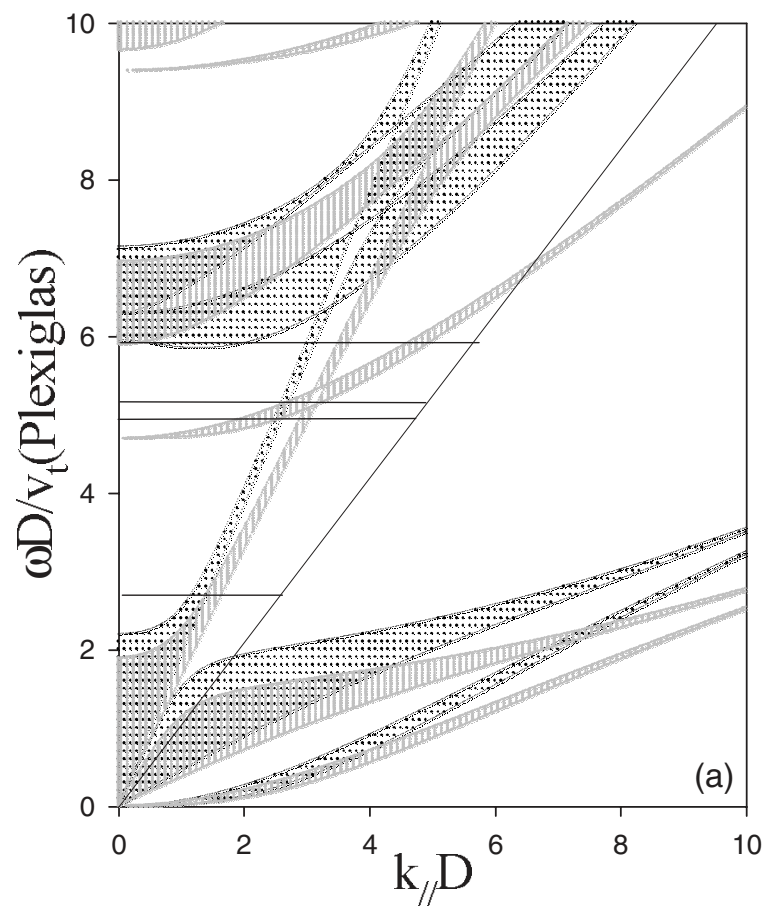

zeros induced by the surrounding solid layers. By decreasing the width of the fluid layer from $d_{0}=1 D$ to $d_{0}=0.6 D$ [Fig. 14(c)], one can notice that the position of the Fano resonance moves to higher frequencies; its width decreases and vanishes for a particular value of $d_{0}=0.71 \mathrm{D}$ before increasing again. At exactly $d_{0}=0.71 D$, the transmission vanishes and the resonance collapses, giving rise to the so-called ghost Fano resonance. ${ }^{58}$ Around $d_{0}=0.71 D$, the asymmetric Fano profile of the resonance becomes symmetric and changes the shape.

In Fig. 14(c), the two solids surrounding the fluid layer have the same widths $d_{s}$; therefore, the transmission zeros induced by the solid layers fall at the same frequency. Now, if the two solids have different widths (labeled for example $d_{s 1}$ and $d_{s 2}$ ), then one can obtain two transmission zeros and a resonance that can be squeezed between these two dips if $d_{s 1}$ and $d_{s 2}$ are chosen appropriately. In this case a symmetric Fano resonance can be obtained whose width can be tuned by adjusting the frequencies of the zeros of transmission. Such resonances have been found also for acoustic and magnetic circuits formed by a guide inserted between two dangling resonators. ${ }^{59,60}$

\section{B. Transmission enhancement assisted by surface resonance}

The possibility of the enhanced transmission from a semiinfinite solid to a semi-infinite fluid, in spite of a large mismatch of their acoustic impedances, has been shown theoretically and experimentally. ${ }^{28}$ The transmission occurs through the surface resonances induced by a 1D solid-solid layered structure inserted between these two media. These reso-

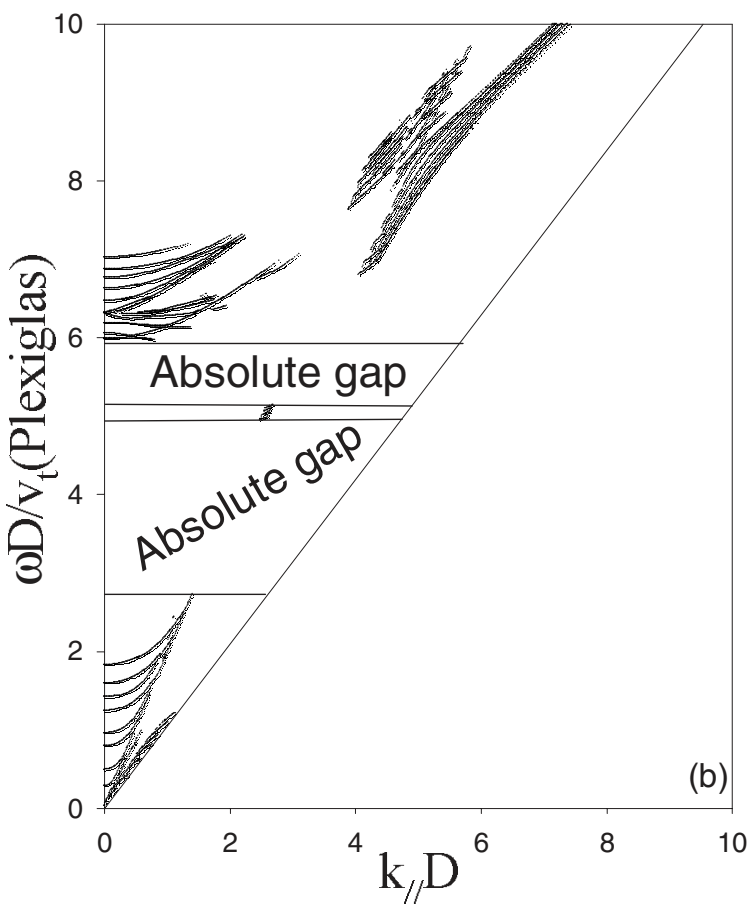

FIG. 13. Band structures for two different SLs, namely, Plexiglas-Hg (dark areas) and PVC-Hg (gray areas). The thickness of the layers are considered to be the same: $d$ (Plexiglas $)=d(\mathrm{PVC})=d(\mathrm{Hg})=D / 2$. (b) Dispersion curves for two coupled finite SLs structures made of $N_{1}=4$ layers of Plexiglas and $N_{2}=4$ layers of PVC immersed in $\mathrm{Hg}$. The discrete modes are obtained from the maxima of the transmission rate that exceeds a threshold of $10^{-3}$. The straight line indicates the $\mathrm{Hg}$ sound line. 


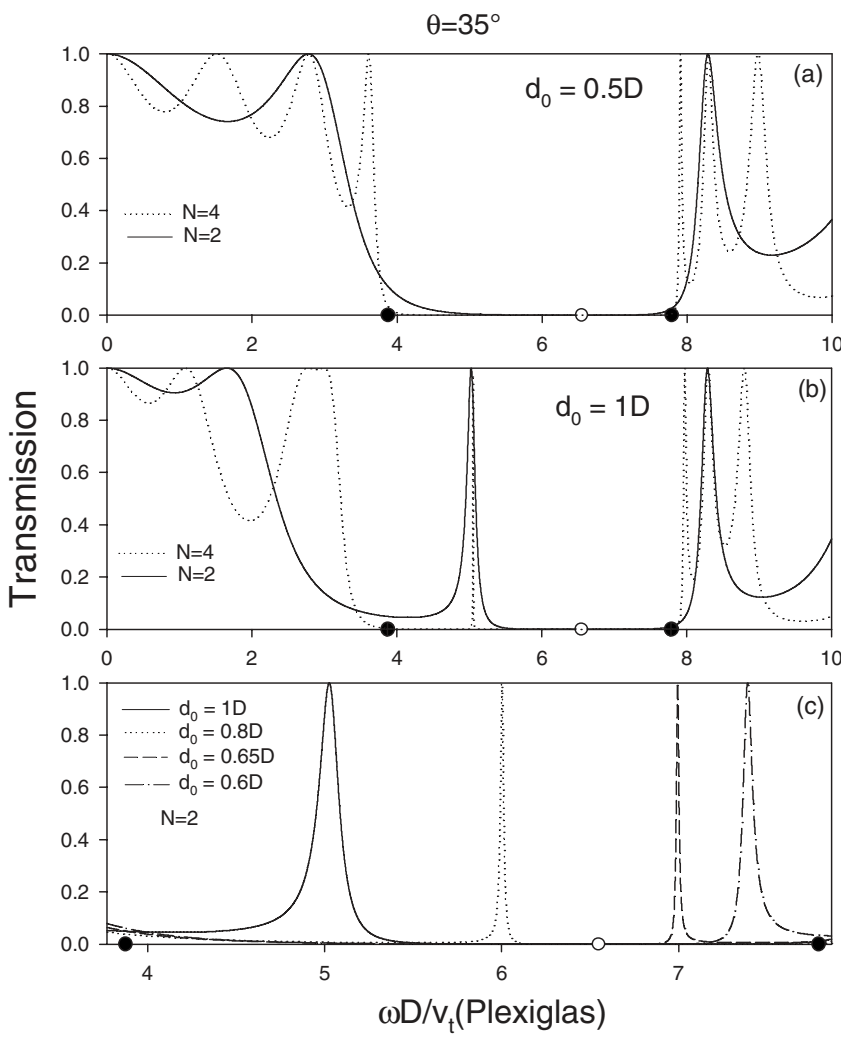

FIG. 14. (a) Transmission rate for a finite SL composed of $N$ $=2$ (solid curves) and $N=4$ (dotted curves) Plexiglas layers immersed in water at an incidence angle $\theta=35^{\circ}$. The solid and open circles on the abscissa indicate the positions of the band-gap edges and transmission zeros, respectively. (b) Same as in (a) but in presence of a defect fluid layer of thickness $d_{0}=D$ at the middle of the structure [see the structure in Fig. 1(e)]. (c) Same as in (b) for $N$ $=2$ and different values of the thickness $d_{0}$ of the cavity fluid layer as indicated in the inset.

nances are attributed to the SL/fluid interface ${ }^{28}$ and coincide with the surface modes of the semi-infinite SL terminated with the layer having the lower acoustic impedance. ${ }^{4}$ Recently, ${ }^{61}$ the possibility of the so-called extraordinary acoustic transmission assisted by surface resonances between two fluids has been shown. The structure consists in separating the two fluids by a rigid film flanked on both sides by finite arrays of grooves. The transmission followed by a strong collimation of sound arises through a single hole perforated in the film.

By analogy with the previous works on this subject, ${ }^{28}$ we show the possibility of enhanced transmission between two fluids by inserting a solid-fluid layered material between these two fluids. Besides the possibility of selective transmission, this structure enables us, from a practical point of view, to separate the two fluids which are, in general, miscible. We give a simple analytical expression of the effective acoustic impedance of the finite SL that enables us to deduce easily the optimal value $N$ of layers in the SL to reach total transmission. In addition to the amplitude analysis, we study also the behavior of the group delay time around the surface resonances as function of $N$. This study has not been performed before. ${ }^{28}$

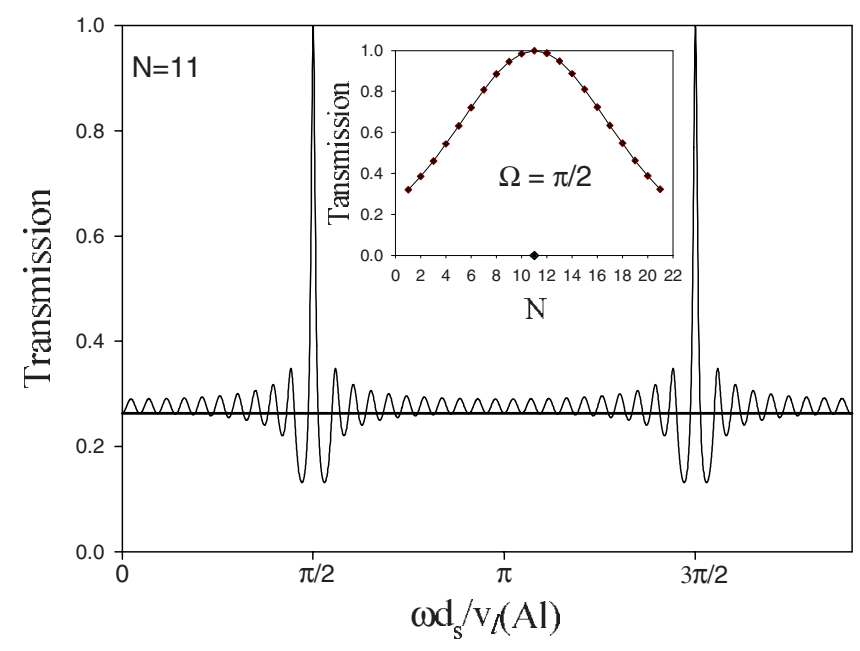

FIG. 15. (Color online) Transmission rate for a finite SL composed of $N=11$ layers of Al separated by $N-1=10$ layers of $\mathrm{Hg}$. The structure is inserted between water (incident medium) and $\mathrm{Hg}$ (detector medium). The inset shows the variation in the maxima of the transmission as a function of the number of unit cells $N$ for the mode situated at $\frac{\omega d_{s}}{v_{\ell}(A l)}=\frac{\pi}{2}$. The straight horizontal line correspond to the transmission rate between water and $\mathrm{Hg}$ (i.e., without the finite SL).

As in the previous works, ${ }^{28}$ we consider a structure formed by a finite solid-fluid SL composed of $N$ solid layers of impedance $Z_{s}$ separated by $N-1$ fluid layers of impedance $Z_{f}$ and inserted between two fluids of impedances $Z_{f 1}$ and $Z_{f 2}$. In the particular case of normal incidence $\left(k_{\|}=0\right)$ and assuming quarter wavelength layers, i.e., $\frac{\omega}{v_{\ell}} d_{s}=\frac{\omega}{v_{f}} d_{f}=\frac{\pi}{2}$, the inverse of the Green's function of the finite SL with free surfaces [Eq. (23)] becomes

$$
g(M M)^{-1}=\left(\begin{array}{cc}
0 & Z_{f}\left(\frac{Z_{s}}{Z_{f}}\right)^{N} \\
Z_{f}\left(\frac{Z_{s}}{Z_{f}}\right)^{N} & 0
\end{array}\right),
$$

which is equivalent to the inverse Green's function of a quarter wavelength layer with an effective acoustic impedance $\left.Z_{e}=Z_{f}\left(Z_{s}\right)_{f}\right)^{N}$. Then we can use the well-known relation ${ }^{2}$ that enables us to use an intermediate layer to form an antireflection coating between two different semi-infinite media, namely, $Z_{f 1} Z_{f 2}=Z_{e}^{2}$. Then we easily get

$$
N=\frac{1}{2} \frac{\ln \left(\frac{Z_{f 1} Z_{f 2}}{Z_{f}^{2}}\right)}{\ln \left(\frac{Z_{s}}{Z_{f}}\right)} .
$$

This relation requires a suitable choice of the materials in order to get a positive value of $N$ greater than unity. In particular, the solid and fluid media constituting the SL should have close impedances.

An example is illustrated in Fig. 15 for a SL composed of $\mathrm{Al}$ and $\mathrm{Hg}$ and sandwiched between water (incident medium) and $\mathrm{Hg}$ (detector medium). The thicknesses of the layers in 


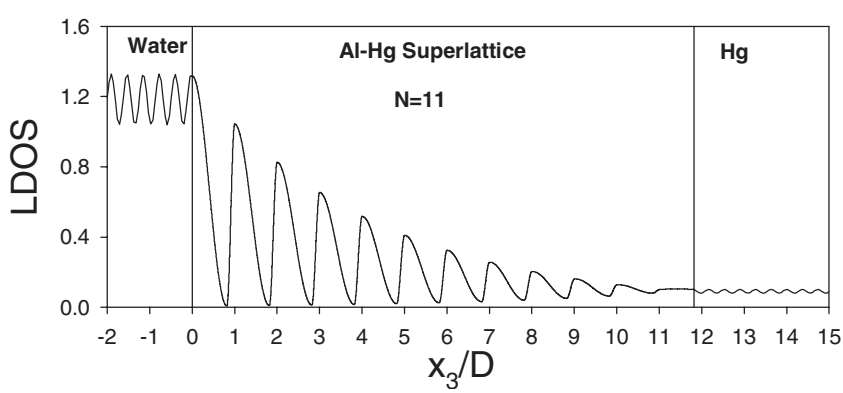

FIG. 16. Variation in the local density of states (LDOS) (in arbitrary units) as a function of the space position $x_{3} / D$ for the surface resonance situated at $\frac{\omega d_{s}}{v_{\ell}(A l)}=\frac{\pi}{2}$ in Fig. 15 and for $N=11$.

the SL are chosen such that $\frac{d_{s}}{v_{\ell}}=\frac{d_{f}}{v_{f}}$. One can see clearly that selective transmission occurs around the reduced frequency $\Omega_{0}=\frac{\omega d_{s}}{v_{\ell}}=\frac{\omega d_{f}}{v_{f}}=(2 n+1) \frac{\pi}{2}$ for a number of cells such that $N$ $=11$ according to Eq. (86). Far from $N=11$, the transmission vanishes as it is illustrated in the inset of Fig. 15. As a matter of comparison, we have also sketched by horizontal line the transmission rate between water and $\mathrm{Hg}$ in the absence of the finite SL. The resonances in Fig. 15 are of Breit-Wigner type $^{28}$ with a Lorentzian shape because of the absence of transmission zeros at normal incidence. Zhao et al. ${ }^{28}$ attributed the resonances lying in the middle of the gaps of the SL to the interference effect of acoustic waves reflected from all periodically aligned interfaces. This explanation is of course correct but a physical interpretation is still needed. We show that the resonances are actually surface resonances induced by the interface between the SL and water. Indeed, after some algebraic calculations, the dispersion relation giving the surface modes [Eq. (81)] of a SL ended with a solid layer in contact with vacuum becomes simply $C_{f}=C_{\ell}=0$, where $C_{f}$ and $C_{\ell}$ are given by Eqs. (9) and (13), respectively. Therefore, one obtains surface modes for

$$
\Omega_{0}=\frac{\omega d_{s}}{v_{\ell}}=\frac{\omega d_{f}}{v_{f}}=(2 n+1) \frac{\pi}{2} .
$$

In addition to Eq. (87), the supplementary condition Eq. (80) that ensures the decaying of surface modes from the surface becomes

$$
Z_{s}<Z_{f}
$$

This condition is fulfilled in the case of a SL made of $\mathrm{Al}-\mathrm{Hg}$. Now, when the Al layer of the SL is in contact with water (instead of vacuum), this latter medium does not affect considerably the position of the surface resonances as the impedance of water is much smaller than Al. In order to confirm the above analysis, we have also sketched the local density of states (LDOS) as a function of the space position $x_{3}$ (Fig. 16) for the mode lying at $\Omega_{0}=\pi / 2$. This figure clearly shows that this resonance is localized at the surface of the SL and decreases inside its bulk. Let us notice that the LDOS reflects the square modulus of the displacement field. Therefore, these results show without ambiguity that the transmission is enhanced by surface resonances.

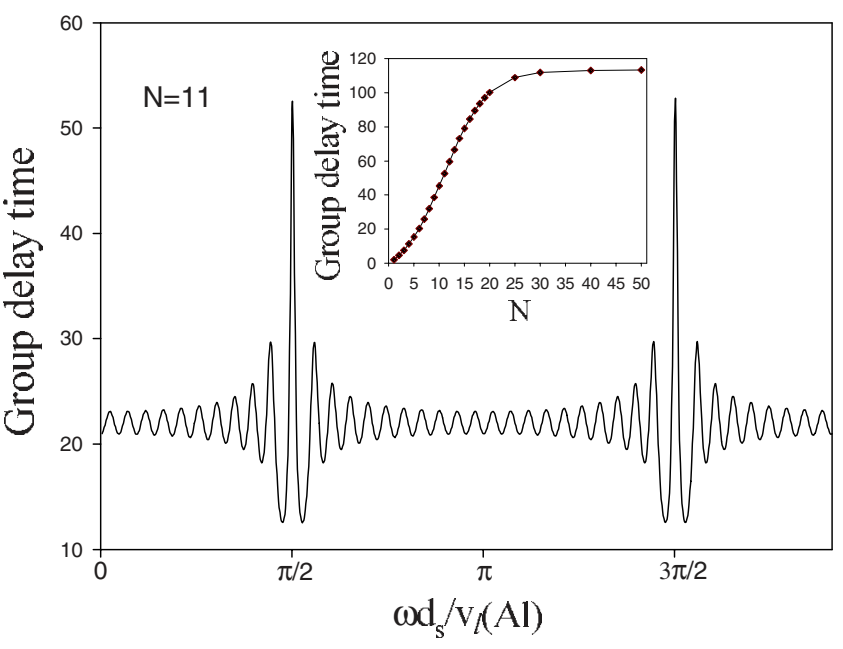

FIG. 17. (Color online) Same as in Fig. 15, but for the transmission delay time [in units of $\left.d_{s} / v_{\ell}(A l)\right]$.

Besides the amplitude of the transmission, we have also analyzed the behavior of the group delay time (Fig. 17). One can notice a strong delay time at the frequencies corresponding to surface resonances, reflecting the time spent by the phonon at the SL/water interface before its transmission. Contrary to the amplitude (see the inset of Fig. 15), the delay time at the surface resonance goes asymptotically to a limiting value $(\sim 110)$ [in units of $\left.d_{s} / v_{\ell}(A l)\right]$ when $N$ increases. This result known as the Hartman effect ${ }^{62}$ arises for classical waves tunneling through a barrier where the delay time saturates to a constant value for a sufficiently barrier's thickness. This phenomenon has been observed experimentally ${ }^{63}$ and explained theoretically ${ }^{64}$ in 1D photonic crystals. For a frequency lying in the allowed bands, the delay time (not shown here) increases linearly as a function of $N$.

The above results can be explained in terms of the DOS. Indeed, due to the similarity between the DOS and the group delay time (Sec. II F), Fig. 17 reflects also the DOS where the different resonant modes are enlarged because of their interaction with the bulk waves of the surrounding media. When $N$ increases, the number of oscillations in the bulk bands (which is related to the number of cells in the system) and the corresponding DOS increase. However, the behavior is different for the peak associated to the surface resonance. Indeed, for low values of $N$, the localization of this mode increases as a function of $N$ because the mode interacts less with the second substrate. So, its width decreases and its maximum increases to ensure an area equal to unity under the resonance peak. However, the peak width cannot decrease indefinitely and reaches a threshold because of its interaction with the first substrate. Therefore, the DOS (or the delay time) saturates to a constant value. By using Eq. (57), we have also examined the group velocity $v_{g}$ in such structures and found that $v_{g}$ oscillates around the mean velocity $v_{m}=D\left(d_{f} / v_{f}+d_{s} / v_{\ell}\right)^{-1}$ inside the bands, whereas this quantity is strongly reduced around the surface resonance. Therefore, such structures can be used as a tool to reduce the speed of wave propagation.

As a matter of completeness we have also checked two other cases: (i) The case where there is no surface resonance 
in the gap of the SL. This can be obtained by using $\mathrm{Hg}$ on both sides of the structure. In this case, even if Eqs. (87) and (88) are satisfied, Eq. (86) gives inacceptable value of $N$ $(N<0)$. In spite of the absence of surface resonances, the delay time saturates to a constant value $(\sim 17)$ (in units of $\left.d_{s} / v_{\ell}(A l)\right)$ at the midgap frequencies because of the Hartman effect. ${ }^{62,64}$ This value is much smaller than in the presence of a surface resonance: (ii) The case where there is two surface resonances in the gap of the SL. This can be obtained by using water on both sides of the structure. In this case, Eqs. (87) and (88) are satisfied and Eq. (86) gives $N \simeq 22$. Because of the existence of two symmetrical surfaces that can support surface modes, one obtains a large surface resonance at $\Omega_{0}=\pi / 2,3 \pi / 2, \ldots$ for $N=22$. For smaller values of $N$, this resonance splits into two distinguished resonances around $\Omega_{0}$ because of the interaction between the two surfaces. A total transmission is still obtained at each resonance. On the contrary, for higher values of $N(N>22)$, there is a single peak in the transmission because the two surface resonances become decoupled, although being enlarged due to their interaction with the substrates. In this case, the transmission peak decreases as far as $N$ increases.

\section{SUMMARY AND CONCLUSION}

In this paper we have presented a comprehensive theoretical analysis of the propagation of sagittal acoustic waves in finite SLs made of alternating elastic solid and ideal fluid layers. We have developed theoretically the expressions giving the Green's functions of different solid-fluid layered media, which enables us to deduce analytically in a closed form the expressions of the dispersion relations, the transmission and reflection coefficients, and the density of states. We have shown analytically and numerically particular features of wave propagation in solid-fluid layered media in comparison with their counterparts composed only of solid media. The main features of solid-fluid SLs is the existence of transmission zeros that are without analog in solid-solid SLs. These transmission zeros exist only for a range of incident angles $\theta$ such that $0<\theta<\theta_{\mathrm{cr}}$. The consequences of the transmission zeros are: (i) The existence of new gaps besides the gaps induced by the periodicity of the system (Bragg gaps). The imaginary part of the Bloch wave vector inside the former gaps is much higher than those in the latter ones, which enables a strong localization of the waves when a defect is inserted in the system; (ii) Besides the closing of the gaps, the solid-fluid SLs present a closing of the gaps, leading to flat bands for which the group velocity vanishes; (iii) The phase of the transmission exhibits a phase drop of $\pi$ and, therefore, a negative delay time or equivalently a negative group velocity, the so-called superluminal phenomena; (iv) The evidence of Fano-type resonances induced by a fluid layer at the vicinity of the transmission zeros induced by the solid layers; and ( $\mathrm{v}$ ) the possibility of the existence of Brewster acoustic angle in the velocity region between transverse and longitudinal velocities of sound in the solid. Total transmission occurs through such angles with mode conversion from longitudinal waves in the fluid to transverse waves in the solid and vice versa. These angles can have practical applications in the area of ultrasonic nondestructive evaluation.

Besides these new properties specific to solid-fluid systems, we have derived exact relations between the density of states and the group delay times in finite systems embedded between two fluids. Also, we have presented a theoretical evidence of the existence of two types of modes in a finite solid-fluid SL made of $N$ cells with free surfaces. In particular, we have shown the existence of $N-1$ modes that fall inside the bulk bands and one additional mode by gap that is associated to one of the two surfaces surrounding the structure. These surface modes are independent of $N$ and coincide with the surface modes of two complementary semi-infinite SLs obtained from the cleavage of an infinite SL between the solid and fluid layers. We have also developed the idea that the solid-fluid layered media exhibit an omnidirectional reflection band gap analogous to the case of 2D and 3D phononic crystals. This property is not fulfilled for incident waves launched from most fluids in contact with the SL. In order to overcome this limitation, we proposed two solutions to realize the omnidirectional mirror, namely, by cladding of the SL with a homogeneous layer, characterized by high velocities of sound, that acts like a barrier for the propagation of phonons or a combination in tandem of two different SLs in such a way that their band structures do not overlap over a given frequency range.

Finally, we have shown two possibilities of enhanced transmission between two fluids. (i) The first solution consists to insert a cavity fluid layer inside the perfect SL. We have evidenced that a simple structure as small as a solidfluid-solid sandwich can exhibit a large gap with sharp resonances of Fano type. This is due to an internal resonance induced by the fluid layer when it falls at the vicinity of the transmission zeros induced by the solid layers. (ii) The second solution consists to insert a finite solid-fluid SL between the two fluids. An effective acoustic impedance of the SL has been derived, which enables to deduce the optimal value of the number $N$ of cells needed to reach the antireflection coating. This occurs around some specific frequencies close to the free surface modes lying in the midgap of the SL, the so-called surface resonances. Contrary to the amplitude, the delay time around these resonances increases monotonically as function of $N$ before saturating at a constant value for a large value of $N$. This phenomenon known as the Hartman effect arises in evanescent regions where waves are traveling by tunneling effect.

We think that most of the analytical expressions given in this manuscript can be used by any reader interested in the subject without going into details of the calculations, and the experimental verification of most of the phenomena predicted in this work can be possible with ultrasonic experiments. $^{34-37}$

\section{ACKNOWLEDGMENTS}

Y.E.H. and E.H.E.B. gratefully acknowledge the hospitality of the Institut d'Electronique, de Microélectronique et de Nanotechnologie (IEMN), UMR CNRS 8520, and UFR de Physique, Université des Sciences et Technologies de Lille. 
*Corresponding author. elboudouti@yahoo.fr

${ }^{1}$ L. M. Brekhovskikh, Waves in Layered Media (Academic, New York, 1981)

${ }^{2} \mathrm{P}$. Yeh, Optical Waves in Layered Media (Wiley, New York, 1988).

${ }^{3}$ E. H. El Boudouti, B. Djafari-Rouhani, E. M. Khourdifi, and L. Dobrzynski, Phys. Rev. B 48, 10987 (1993); E. H. El Boudouti, B. Djafari-Rouhani, A. Akjouj, and L. Dobrzynski, ibid. 54, 14728 (1996).

${ }^{4}$ B. Djafari-Rouhani, L. Dobrzynski, O. Hardouin Duparc, R. E. Camley, and A. A. Maradudin, Phys. Rev. B 28, 1711 (1983).

${ }^{5}$ T. Aono and S. I. Tamura, Phys. Rev. B 58, 4838 (1998).

${ }^{6}$ S. Mizuno and S. I. Tamura, Phys. Rev. B 53, 4549 (1996).

${ }^{7}$ M. Hammouchi, E. H. El Boudouti, A. Nougaoui, B. DjafariRouhani, M. L. H. Lahlaouti, A. Akjouj, and L. Dobrzynski, Phys. Rev. B 59, 1999 (1999); E. H. El Boudouti and B. DjafariRouhani, ibid. 49, 4586 (1994).

${ }^{8}$ H. J. Trodahl, P. V. Santos, G. V. M. Williams, and A. Bittar, Phys. Rev. B 40, 8577 (1989).

${ }^{9}$ W. Chen, Y. Lu, H. J. Maris, and G. Xiao, Phys. Rev. B 50, 14506 (1994).

${ }^{10}$ B. Perrin, B. Bonello, J. C. Jeannet, and E. Romatet, Physica B 219-220, 681 (1996); B. Bonello, B. Perrin, E. Romatet, and J. C. Jeannet, Ultrasonics 35, 223 (1997).

${ }^{11}$ N.-W. Pu and J. Bokor, Phys. Rev. Lett. 91, 076101 (2003); N.-W. Pu, Phys. Rev. B 72, 115428 (2005).

${ }^{12}$ E. M. Khourdifi and B. Djafari-Rouhani, Surf. Sci. 211-212, 361 (1989).

${ }^{13}$ D. Bria, E. H. El Boudouti, A. Nougaoui, B. Djafari-Rouhani, and V. R. Velasco, Phys. Rev. B 60, 2505 (1999).

${ }^{14}$ E. M. Khourdifi and B. Djafari-Rouhani, J. Phys.: Condens. Matter 1, 7543 (1989).

${ }^{15}$ D. Bria, E. H. El Boudouti, A. Nougaoui, B. Djafari-Rouhani, and V. R. Velasco, Phys. Rev. B 61, 15858 (2000).

${ }^{16}$ K.-Q. Chen, X.-H. Wang, and B.-Y. Gu, Phys. Rev. B 61, 12075 (2000).

${ }^{17}$ S. Mizuno, Phys. Rev. B 65, 193302 (2002).

${ }^{18}$ M. Giehler, T. Ruf, M. Cardona, and K. Ploog, Phys. Rev. B 55, 7124 (1997); M. F. Pascual Winter, A. Fainstein, M. Trigo, T. Eckhause, R. Merlin, A. Cho, and J. Chen, ibid. 71, 085305 (2005).

${ }^{19}$ S. I. Tamura, H. Watanabe, and T. Kawasaki, Phys. Rev. B 72, 165306 (2005).

${ }^{20}$ See for example: M. S. Kushwaha, P. Halevi, L. Dobrzynski, and B. Djafari-Rouhani, Phys. Rev. Lett. 71, 2022 (1993); M. Sigalas and E. N. Economou, Solid State Commun. 86, 141 (1993); F. R. Montero de Espinosa, E. Jimenez, and M. Torres, Phys. Rev. Lett. 80, 1208 (1998); J. O. Vasseur, P. A. Dymier, G. Frantziskonis, G. Hong, B. Djafari-Rouhani, and L. Dobrzynski, J. Phys.: Condens. Matter 10, 6051 (1998); I. E. Psarobas, N. Stefanou, and A. Modinos, Phys. Rev. B 62, 278 (2000); 62, 5536 (2000); S. Yang, J. H. Page, Z. Liu, M. L. Cowan, C. T. Chan, and P. Sheng, Phys. Rev. Lett. 88, 104301 (2002).

${ }^{21}$ M. Maldovan and E. L. Thomas, Appl. Phys. Lett. 88, 251907 (2006); P. Dainese, P. S. J. Russel, N. Joly, J. C. Knight, G. S. Wiederheker, H. L. Fragnito, V. Laude, and A. Khelif, Nat. Phys. 2, 388 (2006).

${ }^{22}$ A. Bousfia, E. H. El Boudouti, B. Djafari-Rouhani, D. Bria, A. Nougaoui, and V. R. Velasco, Surf. Sci. 482-485, 1175 (2001); D. Bria, B. Djafari-Rouhani, A. Bousfia, E. H. El Boudouti, and
A. Nougaoui, Europhys. Lett. 55, 841 (2001); D. Bria and B. Djafari-Rouhani, Phys. Rev. E 66, 056609 (2002).

${ }^{23}$ B. Manzanares-Martinez, J. Sanchez-Dehesa, A. Hakansson, F. Cervera, and F. Ramos-Mendieta, Appl. Phys. Lett. 85, 154 (2004).

${ }^{24}$ G. Wang, D. Yu, J. Wen, Y. Liu, and X. Wen, Phys. Lett. A 327, 512 (2004).

${ }^{25}$ M. Trigo, A. Bruchhausen, A. Fainstein, B. Jusserand, and V. Thierry-Mieg, Phys. Rev. Lett. 89, 227402 (2002); P. Lacharmoise, A. Fainstein, B. Jusserand, and V. Thierry-Mieg, Appl. Phys. Lett. 84, 3274 (2004); N. D. Lanzillotti Kimura, A. Fainstein, and B. Jusserand, Phys. Rev. B 71, 041305(R) (2005).

${ }^{26}$ A. J. Kent, R. N. Kini, N. M. Stanton, M. Henini, B. A. Glavin, V. A. Kochelap, and T. L. Linnik, Phys. Rev. Lett. 96, 215504 (2006).

${ }^{27}$ A. Bousfia, Ph.D. thesis, University Mohamed I, 2004.

${ }^{28}$ H. Kato, Phys. Rev. B 59, 11136 (1999); S. Mizuno, ibid. 63, 035301 (2000); D. Zhao, W. Wang, Z. Liu, J. Shi, and W. Wen, Physica B 390, 159 (2007).

${ }^{29}$ S. M. Rytov, Sov. Phys. Acoust. 2, 68 (1956).

${ }^{30}$ M. Schoenberg, Wave Motion 6, 303 (1984).

${ }^{31}$ M. Rousseau, J. Acoust. Soc. Am. 86, 2369 (1989).

${ }^{32}$ M. A. Biot, J. Acoust. Soc. Am. 28, 168 (1956)

${ }^{33}$ B. Gurevich, J. Acoust. Soc. Am. 106, 57 (1999); Geophysics 67, 264 (2002); R. Ciz, E. H. Saenger, and B. Gurevich, J. Acoust. Soc. Am. 120, 642 (2006).

${ }^{34}$ T. J. Plona, K. W. Winkler, and M. Schoenberg, J. Acoust. Soc. Am. 81, 1227 (1987)

${ }^{35}$ C. Gazanhes and J. Sageloli, Acustica 81, 221 (1995).

${ }^{36}$ R. James, S. M. Woodley, C. M. Dyer, and F. Humphrey, J. Acoust. Soc. Am. 97, 2041 (1995).

${ }^{37}$ M. Shen and W. Cao, Appl. Phys. Lett. 75, 3713 (1999).

${ }^{38}$ H. Sanchis-Alepuz, Y. A. Kosevich, and J. Sánchez-Dehesa, Phys. Rev. Lett. 98, 134301 (2007).

${ }^{39}$ E. E. Mendez, F. Agullo-Rueda, and J. M. Hong, Phys. Rev. Lett. 60, 2426 (1988).

${ }^{40}$ Y. El Hassouani, E. H. El Boudouti, B. Djafari-Rouhani, H. Aynaou, and L. Dobrzynski, Phys. Rev. B 74, 144306 (2006).

${ }^{41}$ L. Dobrzynski, Surf. Sci. Rep. 11, 139 (1990)

${ }^{42}$ L. Dobrzynski and H. Puszkarski, J. Phys.: Condens. Matter 1, 1239 (1989).

${ }^{43}$ L. Dobrzynski, J. Mendialdua, A. Rodriguez, S. Bolibo, and M. More, J. Phys. (France) 50, 2563 (1989).

${ }^{44}$ M. Büttiker and R. Landauer, Phys. Rev. Lett. 49, 1739 (1982); M. Büttiker, Phys. Rev. B 27, 6178 (1983); E. H. Hauge and J. A. Stovneng, Rev. Mod. Phys. 61, 917 (1989).

${ }^{45}$ M. L. H. Lahlaouti, A. Akjouj, B. Djafari-Rouhani, L. Dobrzynski, M. Hammouchi, E. H. El Boudouti, A. Nougaoui, and B. Kharbouch, Phys. Rev. B 63, 035312 (2001).

${ }^{46}$ B. Djafari-Rouhani and L. Dobrzynski, J. Phys.: Condens. Matter 5, 8177 (1993).

${ }^{47}$ S. Zhu, N. Liu, H. Zheng, and H. Chen, Opt. Commun. 174, 139 (2000).

${ }^{48}$ B. Manzanares-Martinez and F. Ramos-Mendieta, Phys. Rev. B 61, 12877 (2000).

${ }^{49}$ D. A. Sotiropoulos, J. Sound Vib. 185, 501 (1995).

${ }^{50}$ D. A. Sotiropoulos and R. W. Ogden, Ultrasonics 34, 487 (1996).

${ }^{51}$ E. H. El Boudouti, N. Fettouhi, A. Akjouj, B. Djafari-Rouhani, A. Mir, J. Vasseur, L. Dobrzynski, and J. Zammouri, J. Appl. 
Phys. 95, 1102 (2004)

${ }^{52}$ W. M. Robertson, J. Pappafotis, and P. Flannigan, Appl. Phys. Lett. 90, 014102 (2007).

${ }^{53}$ S.-Y. Ren and Y.-C. Chang, Phys. Rev. B 75, 212301 (2007).

${ }^{54}$ E. H. El Boudouti, Y. El Hassouani, B. Djafari-Rouhani, and H. Aynaou, Phys. Rev. E 76, 026607 (2007).

${ }^{55}$ U. Fano, Phys. Rev. 124, 1866 (1961).

${ }^{56}$ Z. Liu, X. Zhang, Y. Mao, Y. Y. Zhu, Z. Yang, C. T. Chan, and P. Sheng, Science 289, 1734 (2000).

${ }^{57}$ C. Goffaux, J. Sánchez-Dehesa, A. L. Yeyati, P. Lambin, A. Khelif, J. O. Vasseur, and B. Djafari-Rouhani, Phys. Rev. Lett. 88, 225502 (2002); Z. Liu, C. T. Chan, and P. Sheng, Phys. Rev. B 71, 014103 (2005); Y. A. Kosevich, C. Goffaux, and J. SánchezDehesa, ibid. 74, 012301 (2006).

${ }^{58}$ M. L. Ladrón de Guevara, F. Claro, and P. A. Orellana, Phys.
Rev. B 67, 195335 (2003).

${ }^{59}$ E. H. El Boudouti, T. Mrabti, H. Al-Wahsh, B. Djafari-Rouhani, A. Akjouj, and L. Dobrzynski, J. Phys.: Condens. Matter 20, 255212 (2008).

${ }^{60}$ H. Al-Wahsh, E. H. El Boudouti, B. Djafari-Rouhani, A. Akjouj, T. Mrabti, and L. Dobrzynski, Phys. Rev. B 78, 075401 (2008).

${ }^{61}$ J. Christensen, A. I. Fernandez-Dominguez, F. de Leon-Perez, L. Martin-Moreno, and F. J. Garcia-Vidal, Nat. Phys. 3, 851 (2007).

${ }^{62}$ T. E. Hartman, J. Appl. Phys. 33, 3427 (1962).

${ }^{63}$ Ch. Spielmann, R. Szipöcs, A. Stingl, and F. Krausz, Phys. Rev. Lett. 73, 2308 (1994).

${ }^{64}$ P. Pereyra, Phys. Rev. Lett. 84, 1772 (2000); S. Esposito, Phys. Rev. E 64, 026609 (2001); G. Nimtz and A. A. Stahlhofen, Ann. Phys. 17, 374 (2008). 